\title{
Mixing periodic topographies and structural patterns on silicon surfaces mediated by ultrafast photoexcited charge carriers
}

\author{
Jean-Philippe Colombier $\odot,{ }^{1,}{ }^{*}$ Anton Rudenko $\odot,{ }^{1}$ Elena Silaeva $\odot,{ }^{1}$ Hao Zhang, ${ }^{1}$ Xxx Sedao $\odot,{ }^{1}$ Emile Bévillon, ${ }^{1}$ \\ Stéphanie Reynaud, ${ }^{1}$ Claire Maurice, ${ }^{2}$ Florent Pigeon, ${ }^{1}$ Florence Garrelie, ${ }^{1}$ and Razvan Stoian ${ }^{1}$ \\ ${ }^{1}$ Univ Lyon, UJM-St-Etienne, CNRS, Institute of Optics Graduate School, Laboratoire Hubert Curien UMR 5516, \\ F-42023 Saint-Etienne, France \\ ${ }^{2}$ Ecole Nationale Supérieure des Mines de Saint-Etienne, Laboratoire Georges Friedel, CNRS, UMR5307, 42023 St-Etienne, France
}

(Received 12 June 2020; revised 15 September 2020; accepted 22 September 2020; published 15 October 2020)

\begin{abstract}
Ultrafast laser irradiation of silicon can significantly modify charge densities and optical indices, impacting the formation and the development of nanoscale-arranged periodic structures. Photoexcitation degree as well as thermodynamic, hydrodynamic, and structural aspects are reported for crossed orientation of laser-induced periodic surface structures generated on single-crystal silicon after multiple-pulse femtosecond laser irradiation. The periodic topography and microstructure generated by light scattering on surface nanoroughness were characterized to gain insights into the regime of photoexcitation, subsequent thermodynamic conditions, and inhomogeneous energy deposition related to periodic nanostructure formation and growth. A generated free-carrier density around $(3 \pm 2) \times 10^{21} \mathrm{~cm}^{-3}$ is estimated from time-resolved ellipsometry and supported by time-dependent density-functional theory calculations. A finite-difference time-domain solution of the far-field interference of the surface scattered light and the refracted laser wave confirms the periodically crossed energy deposition for this excitation degree. The interference process does not necessarily involve surface plasmon polaritons, and quasicylindrical evanescent waves are identified as plausible scattered waves requiring less restrictive conditions of photoexcitation. Ab initio calculations are also performed to evaluate the transient state of silicon under strong electron-phonon nonequilibrium upon fs laser irradiation. For the reached excitation degree, an electron temperature up to $8000 \mathrm{~K}$ is deduced, supporting local amorphization processes observed as a result of high mechanical stress and quenching rates. A $b$ initio combined with electromagnetic calculations agree well with the results of topography and structural characterization.
\end{abstract}

DOI: 10.1103/PhysRevResearch.2.043080

\section{INTRODUCTION}

Silicon is an important material for electronics and energy applications, and laser structuring offers a promising technique for processing silicon wafers at the micro and nanoscale. Recently, alongside with established operations in trimming, grindings, thinning, dicing, and lithography, the control of the surface topology at the nanoscale has gained in attractiveness [1,2]. In this context, laser-induced periodic surface structures (LIPSSs) carry a certain significance as periodic patterns, scaling below the diffraction limit and responding to local polarization, can perform functions in optoelectronics, biomedical, and mechanical domains [3]. The hierarchical topography has led to the emergence of surface-based design applications in marking, anticounterfeiting, tribology, or wettability [4,5], and in application-driven contexts such as light trapping [6-8], control of surface in reflection $[9,10]$, modifications in contact mechanisms [11],

\footnotetext{
*Email address: jean.philippe.colombier@univ-st-etienne.fr

Published by the American Physical Society under the terms of the Creative Commons Attribution 4.0 International license. Further distribution of this work must maintain attribution to the author(s) and the published article's title, journal citation, and DOI.
}

as well as stimulation and directional growth of living cells [12]. Nonetheless, the formation mechanisms of low spatial frequency LIPSSs (LSFLs) and, especially, high spatial frequency LIPSSs (HSFLs), are still debated, with several interpretations being proposed focusing on either the inhomogeneous energy absorption triggered by electromagnetic scattering [13-16], with a potential role played by a laserinduced oxide layer $[17,18]$ or on the periodic redistribution of the energy by the onset of hydrodynamic waves [19-21]. Whereas the interference between the incident light field and surface scattered waves assisted by evanescent quasicylindrical waves (CWs), surface plasmon polariton (SPP) excitation, and near-field enhancement are supposed to be at the origin of LIPSS formation for metals [14,22-25], semiconductor excitation, turning to a metallic state or not, has received less conclusive explanation [26-28]. As SPPs are not the unique surface waves generated by the interaction of light with nanoreliefs $[25,29]$, the fact that silicon acquires a carrier density corresponding to a metal is questionable. To respond to this question controlling the self-organization phenomenon, it is essential to determine precisely the charge disorder associated to the nonequilibrium state reached by the photoexcited surface.

The interaction of ultrafast laser pulses with a semiconductor surface triggers a combination of linear and multiphoton 
absorption, which transiently determines the onset of a population of free electron-hole pairs. In particular, for near-infrared laser irradiation, silicon undergoes single- and two-photon absorption processes, inducing spontaneously a complex electron-hole plasma state. At fluence levels close to the melting threshold, the thermalization of the excited electrons is assumed to be effective in the first hundred femtoseconds due to the short electron-electron interaction time [30]. As a result, nonequilibrium thermal phenomena occur throughout the free-carrier relaxation with phonons, taking over several picoseconds. Then, depending on the local laser energy and heat loss conditions, phase and structural transitions take place on the surface. The pattern features, in terms of orientation and periodicity of the LIPSS, are known to be mainly defined by the laser wavelength and polarization, suggesting that the early stages of the structure formation can be explained by an electromagnetic approach. The efficacy factor theory developed by Sipe et al. is based on the excitation of an electromagnetic surface wave initiated by random scatter centers that interferes with the incident light field [22]. A periodically enhanced excitation of the material is then generated, depending on the initial roughness topology and the optical properties. A Drude model is frequently combined to this electromagnetic approach to account for the transient optical properties in response to the prior absorbed laser energy via free-carrier excitation [31]. Finite-difference time-domain (FDTD) solutions of this interference resulting from the interaction of laser radiation with the rough surface has extended the Sipe analytical approach, predicting LSFL formation but also HSLF or groove orientation and period [13,21,32]. These structures have been attributed to the inhomogeneous energy absorption evaluated by FDTD calculation, especially when nanoroughness develops and when the amplitude of the topography is enhanced [33]. None of the previous studies has thoroughly addressed the combined effect of the effective excited state of silicon and the structural transformation in regard to the intricate surface morphology.

The purpose of this paper is to evaluate the change disorder in the course of LIPSS formation, taking advantage of the ability of silicon to form simultaneously both HSFLs and LSFLs, depending on the laser irradiation conditions. In an effort to retrace the primary events triggering an inhomogeneous material response from laser absorption to material movement, we focus on understanding oriented conditions where LSFLs and HSFLs are formed with a similar contrast. Combining material characterization techniques and numerical approaches to estimate the degree of nonequilibrium experienced by the irradiated silicon, we experimentally and theoretically address the excited state related to LIPSS formation in these specific conditions. The experimental analysis described in the first section defines the surface nanopatterns associated to the laser conditions by optically probing the transient excited state of irradiated flat-surface silicon. In the calculation part of the paper, the electron excitation level is confirmed by ab initio calculations providing an accurate effective electron density for modeling the inhomogeneous electromagnetic absorption. Measurements, electromagnetic, and ab initio calculations are finally extrapolated to determine the amount of energy sufficient to trigger the observed morphology and local phase transitions in alternative irradiation conditions.

\section{EXPERIMENTAL RESULTS}

\section{A. Microstructural observation of laser-induced crossed patterns}

The LIPSSs on the silicon substrate were produced using a commercially available Ti:sapphire femtosecond laser system (Legend Coherent Inc.). The laser has a central wavelength of $800 \mathrm{~nm}$ with a pulse duration of $60 \mathrm{fs}$ and a repetition rate of $1 \mathrm{kHz}$. Before delivery onto the surface of the silicon sample, the laser pulses were linearly polarized and attenuated through a pair of neutral density filters. A Pockels cell unit is used to control the total number of laser pulses. The laser beam is focused normally, through a $250 \mathrm{~mm}$ achromatic doublet lens onto the sample that is vertically mounted on an X-Y motorized translation stage. The dimension of the beam spot on sample surface, $2 w_{0}=48 \mu \mathrm{m}\left(1 / e^{2}\right.$ intensity), is determined by single shot D-square method [34]. The analysis of surface morphological and microstructural modification in the laser impact area was carried out using a scanning electron microscope (SEM, of Zeiss Supra55 FEG-SEM), equipped with a HKL-Oxford Instruments Electron BackScatter Diffraction (EBSD) system composed of a Nordlys II camera and Channel 5 software suit. EBSD acquisitions were performed at a fixed working distance of $15 \mathrm{~mm}$ and sample tilt of $70^{\circ}$ with respect to horizontal. For EBSD interrogation, the accelerating voltage of primary electron beam was set in the range of $7-30 \mathrm{kV}$, allowing the penetration depth of diffracted backscattered electrons to vary from 5 to $35 \mathrm{~nm}$, approximately [35]. A FEI Helios multisource system with combined functionalities, such as SEM, scanning transmission electron microscopy (STEM), energy dispersive x-ray spectroscopy (EDX), and focused ion beam (FIB) was used for various visualization, analysis, and STEM lamella preparation.

The material irradiated by ultrafast laser pulses in this study was sliced from a pristine single-crystalline silicon (100) wafer. Silicon was chosen not only for its high potential for micro- and optoelectronics and energy technology applications but also for its ability to switch from a crystalline to amorphous phase, depending on local thermodynamic conditions. Structural states as well as HSFL/LSFL formations are expected to be a marker of the local optical index, energy gradients, or phase transitions; all these processes indirectly flowing from the precise density of the photoexcited charge carrier. The laser fluence was chosen such that ablation could happen only in a small central region where the local fluence of the Gaussian beam was beyond the ablation threshold. At a low number of laser pulses, e.g., three, laser ablation created a shallow crater of about $12 \mu \mathrm{m}$ in diameter at the spot center. Within the laser illuminated area but outside the crater, EBSD analysis reveals a concentric annular region of $24 \mu \mathrm{m}$ in diameter and $3 \mu \mathrm{m}$ in width, exhibiting significant lattice damage that is most likely to be silicon amorphization due to transient melting upon ultrafast laser irradiation and subsequent rapid solidification [36,37]. With an increasing number of laser pulses, the crater enlarges while the annular region with lattice damage grows wider inwardly toward the spot center. The laser dose, defining the couple laser fluence and pulse number, were chosen such that LSFLs and HSFLs are both present on the laser impact. 

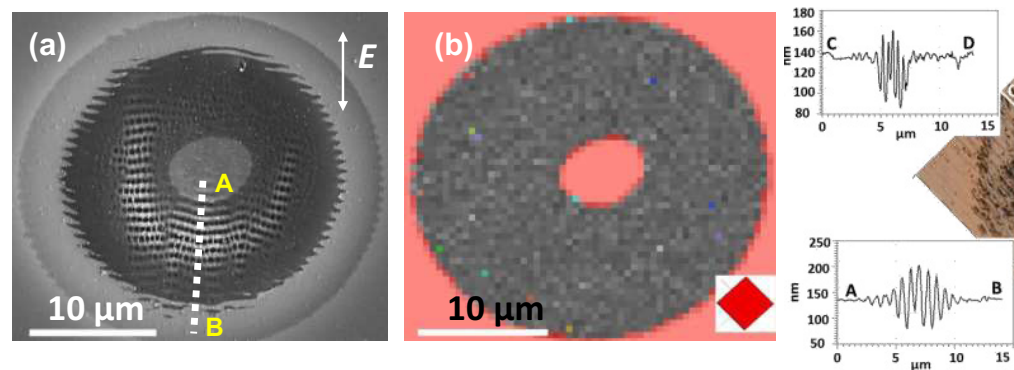

(c)
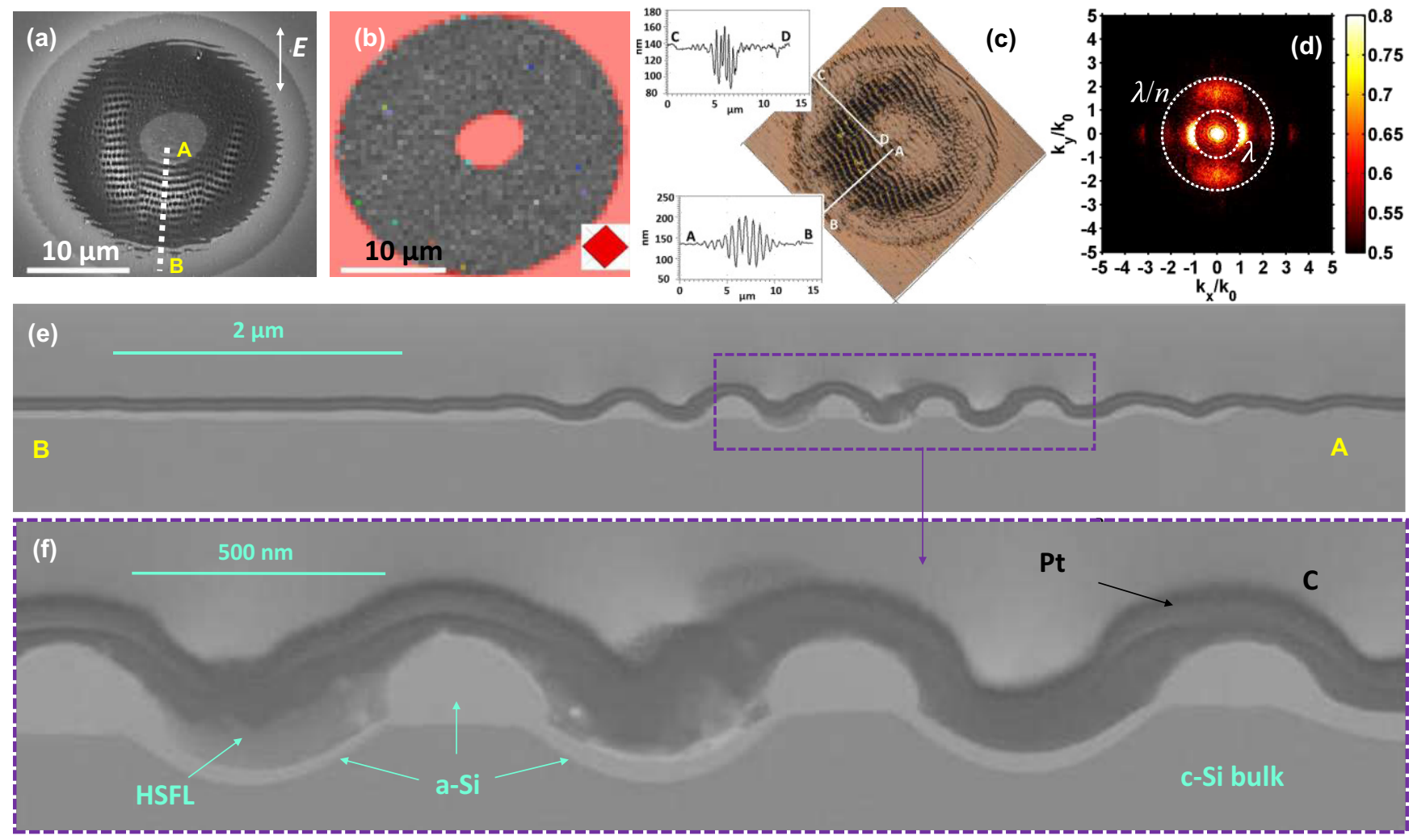

FIG. 1. A site on silicon irradiated by 13 laser pulses of $60 \mathrm{fs}$ at $F_{\text {peak }}=0.27 \mathrm{~J} / \mathrm{cm}^{2}$. (a) SEM image reveals typical features including LSFL, HSFL, ablation crater, and flat zone with contrast. The laser polarization is indicated by the arrow in the upper-right corner. A cross-section lamella (indicated by dashed line AB) is extracted and shown in (e). (b) EBSD map (IPF+BS) shows crystalline state and laser-induced lattice damage. The lower-right corner inset is cube representation of the crystal orientation. (c) AFM topography of the laser impact. Segments AB and CD are indicative of 2D profiles extraction along LSFL and HSFL. The 2D profiles are shown as insets. (d) 2D-Fourier transform of the SEM image with axis normalized by the free-space wave number $k_{0}=2 \pi / \lambda$. The dashed circles represent $|k|=\operatorname{Re}(\tilde{n}) k_{0}$ and $|k|=k_{0}$. (e) STEM images of the cross section illustrate the entire cross section at low magnification and reveal distribution of amorphous silicon. The selected area (indicated by the dashed rectangle) is further magnified and shown below in (f). The carbon and platinum are protective layers deposited before FIB sectioning.

A SEM micrograph highlighting the coexistence of LSFLs and HSFLs inside a laser impact, with the former periodic structure being perpendicular to the laser polarization, and the later one parallel to the polarization, is shown in Fig. 1(a) [3,14]. The sample was made with 13 laser pulses at a fluence of $0.27 \mathrm{~J} / \mathrm{cm}^{2}$. Although a very specific condition is presented here as an example, it is worth noting that within a moderate laser fluence variation range $( \pm 30 \%)$ around $0.27 \mathrm{~J} / \mathrm{cm}^{2}$, the coformation of LSFLs and HSFLs was always observed, at the expense of fewer or more laser pulses. Outside this process window, for instance, at lower laser fluence, i.e., $0.1 \mathrm{~J} / \mathrm{cm}^{2}$ and less, even many pulses would only cause HSFLs to form. For elevated laser fluence, material removal takes place at small pulse numbers, and ablation crater warps or prevents the periodic structure formation. Also, normal incidence and linear polarization conditions have been used as oblique incidence and sophisticated vectorial polarization states lead to undesired complexity of the light-surface coupling but still local polarization-dependent patterns [38]. In the particular case shown in Fig. 1(a), a few characteristic annular regions appear in the laser impact. In the central disk region of $8 \mu \mathrm{m}$ in diameter, the LSFL with a periodicity $\Lambda_{L} \simeq 760 \mathrm{~nm}$ is the sole morphology present in this otherwise featureless area. Out of the central spot, intertwined LSFLs and HSFLs (periodicity $\Lambda_{H} \simeq 330 \mathrm{~nm}$ ) cover a roughly circular-shaped area of $20 \mu \mathrm{m}$ in diameter. This area shows a distinct bright contrast, suggesting sharp changes of surface topography. While spreading out radially, both LSFLs and HSFLs vanish gradually and the surface turns into a zone without distinguishable features which terminates at the rim of the ablation crater. The ablation crater is clearly defined by an aperture rim of $12-\mu \mathrm{m}$ radius. There is another ring-shaped region of $3 \mu \mathrm{m}$ wide outbound the ablation rim with a contrast darker than the silicon surface surrounding the impact. The dashed line $A B$ marks the position where a cross-section lamella was extracted using FIB. The horizontal domain is narrow and corresponds to LSFLs, which is more pronounced than the more diffused vertical one related to HSFLs. Figure 1(b) is an EBSD map [inverse pole figure (IPF) and band slope (BS)] generated at $20 \mathrm{kV}$ that illustrates the lattice state of the laser impact. The central disk area of $8 \mu \mathrm{m}$ in diameter has a high degree of crystallinity with crystallographic orientation identical to that of the substrate (100). A circular region of $26-\mu \mathrm{m}$ diameter outbounds the central disk appearing dark in the EBSD map is characterized by a marked lattice disturbance (the darker the area, the higher degree of lattice defects) [39]. This lattice 
disturbance manifests itself in the form of local amorphization [35], which is spatially resolved in Figs. 1(e) and 1(f). This circular region coincides with the annular regions in Fig. 1(a).

The surface topography changes after laser irradiation was measured using an atomic force microscope (AFM, Agilent 5500) shown in Fig. 1(c). The annular region with LIPSSs appears wavier than the central disk region. To highlight the peak-to-valley amplitude of the various surface features, two 2D surface profiles are extracted in the way indicated by the segments AB (across LSFLs) and CD (across HSFLs) in the figure and displayed as insets. The amplitude of the LSFLs is moderate in the central disk region (around $20 \mathrm{~nm}$ ) and increases up to $120 \mathrm{~nm}$ in the circular region. The amplitude for HSFLs is slightly smaller, about $70 \mathrm{~nm}$ maximum. The step at the rim of the crater measures approximately $5 \mathrm{~nm}$. It is worthwhile to note that the crests of both LSFLs and HSFLs protrude above the initial surface level. The 2D Fourier transform presented in Fig. 1(d) has been performed on the SEM image to emphasize the two kinds of nanostructures and the dispersion on the measured periodicity. The spatial spectrum of LSFLs exhibits a sickle shape with a period slightly lower than $\lambda$ whereas the HSFL spectrum is more spread and is clearly enclosed by the $\lambda / \tilde{n}$ circle where $\tilde{n}$ is the estimated optical index of silicon with $N_{e}=3 \times 10^{21} \mathrm{~cm}^{-3}$ excited electrons in the conduction band as evaluated by the theory developed in Sec. III.

The dark-field STEM images of the cross section are presented in Fig. 1(e). The letters $A B$ are for visual guidance corresponding to the selected site AB in Fig. 1(a). The top layers are carbon and platinum $(\mathrm{Pt})$ protective coatings deposited before the FIB lift-out process. A thin layer with light-grey contrast right beneath the dark Pt layer is pure silicon. The EBSD analysis of the lamella performed in the transmission mode further confirms that this silicon layer is amorphous $(a-\mathrm{Si})$. At the outer region of the laser impact (side B), the interface between $a$-Si and underlying bulk silicon is almost a straight line parallel to the surface. The thickness of the $a$-Si is about $50 \mathrm{~nm}$. The $a$-Si thins out toward the center of the laser impact (side A). The LSFL features in the middle of the crosssection lamella is further enlarged and shown in Fig. 1(f). The $a-\mathrm{Si}$ on the crests is hemisphere shaped while the $a-\mathrm{Si}$ remains thin and uniform in thickness on the shoulders and in the valleys of the LSFL. Interestingly, the interface between amorphous $(a-\mathrm{Si})$ and crystalline silicon $(c-\mathrm{Si})$ in the crests appears plateaulike and the plateau has the same level as the interface at the aforementioned outer area. A marked area is likely to be a partially sectioned HSFL. Note that the EDX analysis suggests a Pt presence in this area which must have been introduced into the zone during the deposition before FIB sectioning.

At this irradiation fluence, the flat topography at the center of the impact coupled with the absence of an amorphous phase formation is a remarkable effect that will be discussed in the last section. The observed phase transitions are spatially distributed with a periodic predominance, most probably related to the temperature level reached locally. These alternating amorphous-crystalline fringes confirm the possibility to initiate local phase transitions by swift local melting at the maximum energy deposition in addition to surface depression [40]. These maxima are intrinsically correlated with the generation and heating of free carriers by electromagnetic energy deposition. At attenuated laser fluence (not shown here), the center of the laser spot becomes amorphous and LIPSSs appear in the center, consolidating both silicon amorphization and the periodic structure formation are highly sensitive to the local fluence value. To elucidate the degree of optical injection of electron-hole pairs into the silicon surface and their subsequent temporal evolution with subpicosecond time resolution, we performed time-resolved reflection experiments described below.

\section{B. Time-resolved dynamics}

In this section, we evaluate the transient free-electron density by pump-probe experiments. The plasma generation in femtosecond photoexcited silicon is resolved in time in conditions similar to LIPSS formation. Ultrafast laser excitation of silicon forms a dense electron-hole plasma of density $N_{e}$, significantly altering the optical response of the free carriers from low excitation values. Neglecting recombination and diffusion processes, a rough estimation of the carrier density generated during the laser pulse can be obtained integrating in time dynamic equations for $N_{e}$ and the pump intensity $I$ [41],

$$
N_{e} \simeq \frac{F\left[1-R\left(N_{e}\right)\right]}{\hbar \omega}\left\{\alpha+\beta \frac{F\left[1-R\left(N_{e}\right)\right]}{2 \sqrt{2} \tau_{\mathrm{FWHM}}}\right\},
$$

where $R\left(N_{e}\right)$ is the reflectivity of the excited surface, $\alpha=$ $1.02 \times 10^{5} \mathrm{~m}^{-1}$ and $\beta=10^{-10} \mathrm{~m} \mathrm{~W}^{-1}$, the one- and twophoton absorption coefficients at the given wavelength $\lambda=$ $800 \mathrm{~nm}$, respectively $[27,31,42,43]$. The impact region where LIPSSs are observed in Fig. 1 corresponds to a local fluence $F \simeq 0.15-0.25 \mathrm{~J} / \mathrm{cm}^{2}$, suggesting a range of electron density of $(2-5) \times 10^{21} \mathrm{~cm}^{-3}$. Under this assumption, the optical response under ultrashort absorption can be captured by a Drude-like free-carrier form, and the permittivity of the excited silicon writes $\varepsilon=\varepsilon_{\infty}-\left(\frac{\omega_{p}}{\omega}\right)^{2} \frac{1}{1+i\left(\omega \tau_{D}\right)^{-1}}$, where $\varepsilon_{\infty}$ is the permittivity of the unexcited material, $\omega$ and $\omega_{p}\left(N_{e}\right)$ are the laser and plasma frequency, respectively, and $\tau_{D}\left(N_{e}\right)$ is the Drude damping time [41]. The static properties of the unexcited silicon surface were evaluated ex situ using a commercial ellipsometer (Uvisel, Horiba Jobin Yvon) and the results match well-known data [44]. The standard value of $\varepsilon_{\infty}=13.6+i 0.048$ at $800 \mathrm{~nm}$ is thus considered in the following for unexcited silicon. Following Fresnel formalism, the reflectivity dependence with excitation is shown in Fig. 2 at low $\left(27.1^{\circ}\right)$ and high $\left(65.8^{\circ}\right)$ incident angles. The highangle curve anticipates large reflectivity changes by up to a factor of 18 between unexcited and excited silicon.

To refine this crude estimation of $(2-5) \times 10^{21} \mathrm{~cm}^{-3}$ free electrons and evaluate more accurately the degree of electron excitation allowing the LIPSS formation, ultrafast pump-probe reflectivity measurements were performed to probe carrier dynamics for relevant intensity values. To pump the sample to an optically excited state, a single $s$-polarized femtosecond laser pulse was focused on the sample surface at normal incidence. The transient optical properties of silicon surfaces upon ultrafast laser irradiation were probed by $p$-polarized probe pulses at the same photon energy using a two-angle time-resolved ellipsometry/reflectometry method as proposed in Ref. [45]. 


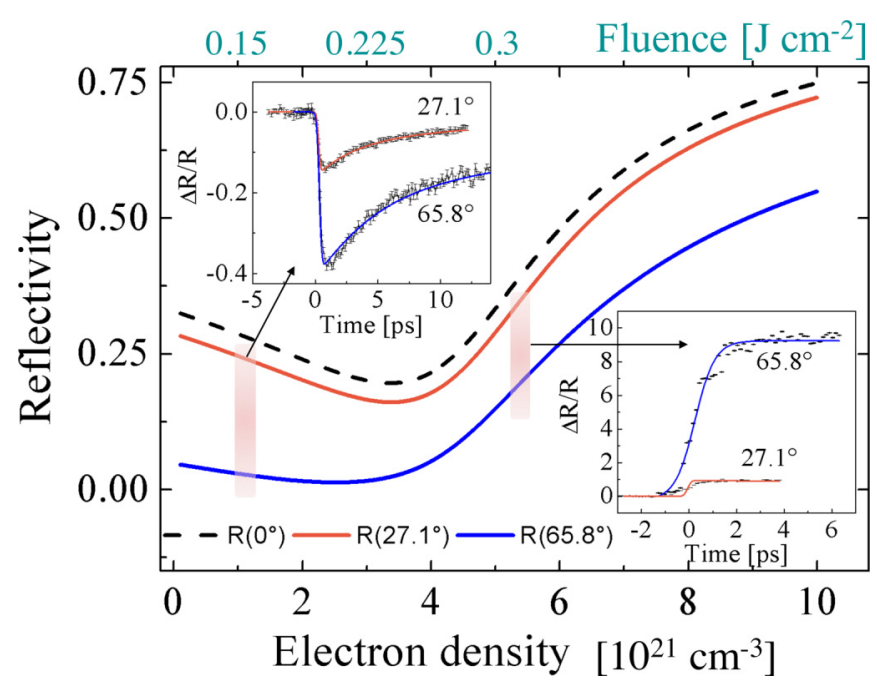

FIG. 2. Theoretical reflectivity of silicon for the pump and probes incident angles $\left(0^{\circ}, 65.8^{\circ}, 27.1^{\circ}\right.$, respectively, as a function of electron density. Insets: Time-resolved $p$ reflectivity changes at two probe angles on silicon $\left(27.1^{\circ}\right.$ and $\left.65.8^{\circ}\right)$ for two fluences, corresponding to two excitation degrees.

Two $p$-polarized probe pulses were time-synchronized with fs accuracy with the exciting pump pulse and were focused on the same spot as the pump pulse but at oblique incidence at $27.1^{\circ}$ and $65.8^{\circ}$ incidence angles. The lens to focus the pump beam has a focal length of $f=30 \mathrm{~cm}$ while the two probe beams are focused by a $f_{1}=5 \mathrm{~cm}$ lens and a $f_{2}=3 \mathrm{~cm}$ lens, respectively. To ensure that the probe pulses only detects the center of the excited region, the focal diameter of the pump pulse is adjusted to be at least four times larger than the probe beam. The exciting peak fluence $I^{p}$ was chosen to set the limit values of the local intensities triggering the LIPSS and microstructural changes, namely, $I_{1}^{p}=5.3 \times$ $10^{12} \mathrm{~W} / \mathrm{cm}^{2}$ and $I_{2}^{p}=2.8 \times 10^{12} \mathrm{~W} / \mathrm{cm}^{2}$, corresponding to $F_{1}=0.315 \mathrm{~J} / \mathrm{cm}^{2}$ and $F_{2}=0.167 \mathrm{~J} / \mathrm{cm}^{2}$, respectively. The transient reflectivity changes were measured by two photodiode detectors in imaging geometries with respect to the surface.

The changes in reflectivity $\Delta R / R$ with pump-probe delay are plotted for the two chosen intensities and the two probe angles as insets on Fig. 2, where expected reflectivity $R$ as a function of the excited free-electron density is also shown. To guide the eyes, the experimental data are fitted by the convolution of the Gaussian envelope of the laser pulse with a linear combination of several exponential functions as the measured signal is a convolution between the pulse duration and the carrier dynamics. To access the evolution of optical indices, we focus on the measured maximum values of the transient reflectivity changes as they represent uniquely determined pairs $\operatorname{Re}(\varepsilon), \operatorname{Im}(\varepsilon)$ which are extracted by inverting Fresnel formulas at the given angles [46,47]. The measured data yields of the excited permittivities give $\varepsilon=-3.35+i 2.16$ at a peak intensity $I_{1}^{p}$ and $\varepsilon=10.41+$ $i 2.30$ for $I_{2}^{p}$. They correspond to the maximum density values reached during the dynamics of the electron-hole plasma generation and from the Drude formalism; we can extract the couples $N_{e}^{1, \max }=5.4 \times 10^{21} \mathrm{~cm}^{-3}$ with $\tau_{D}^{1, \max }=3.4 \mathrm{fs}$ and
$N_{e}^{2, \max }=1.5 \times 10^{21} \mathrm{~cm}^{-3}$ with $\tau_{D}^{2, \max }=0.6$ fs. The range $(1-6) \times 10^{21} \mathrm{~cm}^{-3}$ is thus in full agreement with previously reported estimations of electron densities corresponding to excited optical properties obtained by a Sipe-Drude model which results in spatial electric field response with preferential orientation similar to LSFLs [31]. These two electron density values reflect a photoexcitation process integrated over the probe duration and follows carrier dynamics as shown by the time-resolved measurement of the optical properties. Also, the spatial resolution is limited by the focal spot of the probe beam and necessarily integrates a nonhomogenous intensity distribution. For this reason, two excitation intensities were presented allowing to enclose the expected intensity distribution on the structuring experiment shown in Fig. 1. We observe that the deduced value of density for $I_{1}$ is slightly higher than the critical density $N_{e}^{\text {cr }}=4.91 \times 10^{21} \mathrm{~cm}^{-3}$ and for $I_{2}$ it is lower than this limit value corresponding to the semiconductor-to-metal transition, namely, $\varepsilon_{r}=0$. For $I_{1}$, the optical index even shows a value of $\varepsilon_{r}<-1$ allowing the excitation of SPPs. Given that this value is assumed to represent a maximum value and that the formation of SPP covers a large area of the impact, for which a lower intensity level is expected, it is legitimate to question whether SPP excitation is a sine qua non condition or whether $N_{e}$ lower than the required condition remains a possibility. Therefore, it is necessary to verify whether the energy contained in these carriers is sufficient to generate the phase transitions and matter displacement associated with the observed LIPSS. We propose the following theoretical considerations to clarify these questions.

\section{THEORETICAL CALCULATIONS ON PHOTOEXCITED CHARGE CARRIERS}

To further correlate the measured level of free-carrier excitation to the observed periodic structural topography, theoretical analysis of the expected electronic structure redistribution combined with electromagnetic simulations yields the condition leading to the onset of crossed LIPSS (HSFL and LSFL) formation along with local phase transitions. This has been performed in two successive steps. The first one consists of the determination of the electron density by timedependent density-functional theory (TDDFT) and the second one to the deduction of the corresponding temperature in a stationary approach by density-functional theory (DFT).

\section{A. Estimation of the transient electron density from TDDFT calculations}

The density of laser-excited electrons in silicon is calculated using TDDFT $[48,49]$ as implemented in OCTOPUS code [50]. In this real-time TDDFT, the laser-induced electronexcitation process is described by time-dependent Kohn-Sham equations,

$$
i \hbar \frac{\partial}{\partial t} \psi_{i}(\mathbf{r}, t)=H_{\mathrm{KS}}(\mathbf{r}, t) \psi_{i}(\mathbf{r}, t),
$$

where $\psi_{i}(\mathbf{r}, t)$ is a wave function of a single-electron state, $i$ denotes the state index and runs over all initially occupied 
states. The Kohn-Sham Hamiltonian $H_{\mathrm{KS}}(\mathbf{r}, t)$ is given by

$$
\begin{aligned}
H_{\mathrm{KS}}(\mathbf{r}, t)= & \frac{1}{2 m}\left(-i \nabla+\frac{e}{c} \mathbf{A}(t)\right)^{2} \\
& +v_{\mathrm{ion}}(\mathbf{r})+v_{\mathrm{H}}(\mathbf{r}, t)+v_{\mathrm{xc}}(\mathbf{r}, t) .
\end{aligned}
$$

It includes ionic potential $v_{\text {ion }}(\mathbf{r})$, Hartree potential $v_{\mathrm{H}}(\mathbf{r}, t)$ and exchange-correlation potential $v_{\mathrm{xc}}(\mathbf{r}, t)$. A metageneralized-gradient approximation of Tran and Blaha is employed for the exchange-correlation potential [51]. This potential is known to well reproduce both indirect and direct band gaps of silicon [52]. The Troullier-Martins pseudopotential is used for electron-ion interaction [53]. The ion positions are fixed. The spatially uniform electric field of the laser pulse is introduced through vector potential $\mathbf{A}(t)$,

$$
\mathbf{A}(t)= \begin{cases}-c \frac{E_{\mathrm{m}}}{\omega} \cos (\omega t) \sin ^{2}\left(\pi t / \tau_{\mathrm{L}}\right) & \text { if } 0<t<\tau_{L} \\ 0 & \text { otherwise, }\end{cases}
$$

where $\omega$ is laser frequency and pulse duration $\tau_{\mathrm{L}}=165 \mathrm{fs}$ is chosen to obtain the given $\tau_{\mathrm{FWHM}}=60 \mathrm{fs}$. The peak electric field inside the medium $E_{\mathrm{m}}$ depends on the peak electric field in vacuum $E_{\mathrm{v}}$ as $E_{\mathrm{m}}=2 E_{\mathrm{v}} /(n+1)$ where $n=3.68$ is the real part of the refractive index of unexcited silicon at $800 \mathrm{~nm}$ [44]. The refractive index is assumed to change negligibly at the given excitation level. The peak electric field in vacuum is defined by $E_{\mathrm{v}}=\sqrt{2 I_{\mathrm{v}} /\left(c \varepsilon_{0}\right)}$, where $I_{\mathrm{v}}$ is the peak intensity of laser pulse in vacuum.

In OcTOPus code, Eq. 2 is solved in a real-space threedimensional grid with an enforced time-reversal symmetry method for time propagation. A cubic unit cell with a length of $0.54 \mathrm{~nm}$ and discretized into $16^{3}$ grid points is considered. The unit cell contains eight silicon atoms with four valence electrons each. The $k$ space is discretized into $8^{3} k$ points. The number of $k$ points is limited due to a very long calculation time. An error of about $10 \%$ is, however, expected compared to higher number of $k$ points [48]. A time step of $9.4 \times 10^{-4}$ fs is used.

The density of electrons $N_{e}(t)$ promoted from the valence band into the conduction band is first calculated as follows:

$$
N_{e}(t)=\frac{N_{a}}{8} \sum_{j} f_{j}\left(1-\sum_{i}\left|\left\langle\phi_{j} \mid \psi_{i}(t)\right\rangle\right|^{2}\right),
$$

where $\psi_{i}(t)$ is the solution of Eq. $2, \phi_{j}$ is the ground-state wave functions satisfying the eigenvalue equations: $H_{\mathrm{KS}}^{t=0} \phi_{j}=$ $\epsilon_{j} \phi_{j}, f_{j}$ is the initial occupation number of state $j$ and $N_{a}=$ $5 \times 10^{22} \mathrm{~cm}^{-3}$ is density of silicon atoms. The indices $i, j$ run over occupied states. Then the result of Eq. 5 is corrected by taking into account adiabatic evolution of the ground state due to the evolution of the vector potential $[49,54]$.

Figure 3 shows the calculated density of laser-excited electrons as a function of time $N_{e}(t)$ in silicon. The excitedelectron density of $3.2 \times 10^{21} \mathrm{~cm}^{-3}$ and $1 \times 10^{21} \mathrm{~cm}^{-3}$ is obtained in the end of the laser pulse with fluences $0.25 \mathrm{~J} / \mathrm{cm}^{2}$ and $0.15 \mathrm{~J} / \mathrm{cm}^{2}$, respectively. Such fluences correspond to the peak intensities $4.2 \times 10^{12} \mathrm{~W} / \mathrm{cm}^{2}$ and $2.5 \times 10^{12} \mathrm{~W} / \mathrm{cm}^{2}$ of the 60-fs laser pulse, respectively. After the laser pulse ends, $N_{e}$ stays constant because the recombination mechanisms are not taken into account in this framework. The decrease of the density observed slightly after the maximum of the laser-pulse

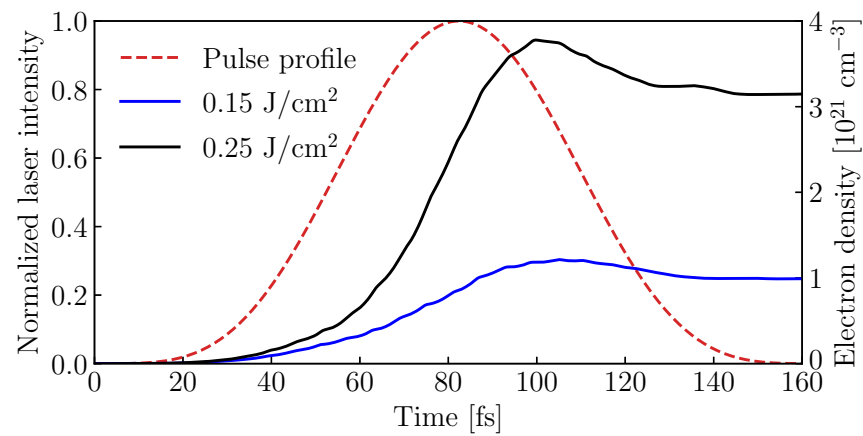

FIG. 3. Evolution of excited electron density in silicon illuminated with a laser pulse of $\tau_{\mathrm{FWHM}}=60 \mathrm{fs}$ and fluence of $0.25 \mathrm{~J} / \mathrm{cm}^{2}$ (black solid line) and $0.15 \mathrm{~J} / \mathrm{cm}^{2}$ (blue solid line). Normalized intensity profile of the laser pulse is shown by a red dashed line.

intensity is related to the virtual excitations of electrons below the silicon direct band edge [55,56]. The virtually excited electrons exist only in the presence of high laser field and do not contribute to the dissipation of energy into material.

\section{B. Estimation of the electron temperature from DFT calculations}

To estimate the temperature of excited free carriers (electrons and holes) after they are mutually thermalized, a static DFT approach is used. The system is kept in a stationary state, not necessarily the ground state but a hot state. Assuming an electron-phonon nonequilibrium state with thermalized electrons at $T_{e}$, the estimated free-electron density provides indirect access to the temperature of the electron subsystem [57]. In a band-gap material, it is often assumed that hole and electron populations rapidly reach distinct thermalized states characterized by two different chemical potentials [58]. However, this implies prior knowledge of the density of free carriers at a given electron temperature; accordingly, we restrain our modeling to a classical single Fermi-Dirac function for the temperature dependent distribution of electrons.

The calculations are carried out with the plane-wave code ABINIT [59] in the frame of the electron TDDFT [60-62]. The exchange and correlation part is modeled within the generalized gradient approximation (GGA) in the form parameterized by Perdew et al. [63]. Projector augmented-wave method [64] is used to model nuclei and core electrons of the two atoms of the silicon primitive cell. A $34 \times 34 \times 34 k$-point grid has been used to mesh the reciprocal space following the Monkhorst $k$-point distribution method [65]. To accommodate the electron temperature used to describe the laser excitation, 30 bands per atom are considered. The convergence with regard to these parameters has been carefully tested. In this calculation with GGA exchange-correlation potential, the obtained band gap $0.61 \mathrm{eV}$ is underestimated. To obtain the experimentally expected value of $1.17 \mathrm{eV}$ [66], we first computed the electron-temperature-dependent density of states (DOS) of silicon. The DOS was found to be remarkably stable against heating, up to very high temperature. Considering such DOS stability at increasing $T_{e}$ and the homogeneous gap underestimation along the reciprocal space [67], we then artificially increased the band gap of the $0 \mathrm{~K}$ DOS to its 

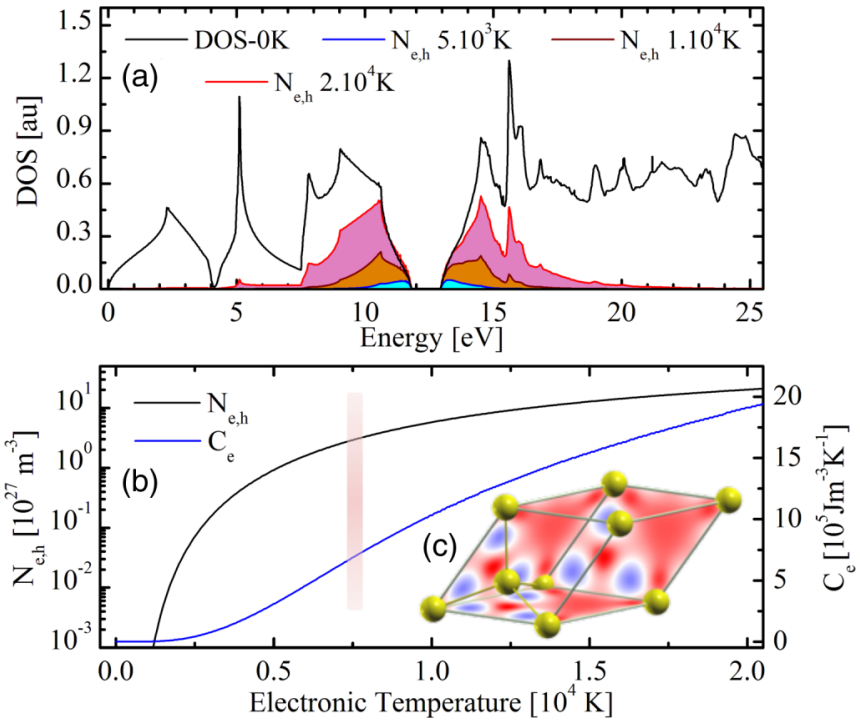

FIG. 4. (a) Density of states of silicon, with a band gap artificially increased to reach the $1.17 \mathrm{eV}$ experimental value. $N_{e}$ corresponds to the magnified proportion of holes and electrons created during the heating process. (b) Electron density $N_{e}$ and heat capacity $C_{e}$ as a function of the electron temperature. (c) Primitive cell of silicon, with the three basal planes (100), (010), and (001) showing the difference between $8000 \mathrm{~K}$ and $0 \mathrm{~K}$ electron density spatial distribution. Blue and red gradients indicate areas of respectively decreasing and increasing electron densities.

experimental value (scissors operation). Electron temperatures are further applied to this modified band structure through the Fermi-Dirac distribution of the electrons by adjusting the electron chemical potential $\mu$ to keep constant the number of valence electrons.

The corresponding DOS and the increasing proportion of holes and electrons created during the heating process are presented in Fig. 4(a). The calculated change of the electron density spatial distribution between $8000 \mathrm{~K}$ and $0 \mathrm{~K}$ is shown in Fig. 4(c). It indicates a displacement of the electron density from the directional $\mathrm{Si}$-Si bonding toward an interatomic nonbonding area, contributing to a weakening of the $\mathrm{Si}-\mathrm{Si}$ bonding, in agreement with previous works [68].

At a given $T_{e}$, the number of conduction electrons $N_{e}$ is deduced from the integration over the conduction band energy $N_{e}\left(T_{e}\right)=\int_{E}^{\infty} g(\epsilon) f\left(\epsilon, \mu\left(T_{e}\right), T_{e}\right) \mathrm{d} \epsilon$ [69]. $N_{e}$ is equal to the number of holes $N_{h}$, which is also equal to the number of electron-hole pairs $N_{e, h}$, assuming electrons and holes are associated. In Fig. 4(b), the evolution of $N_{e}$ with $T_{e}$ is provided, together with the electron heat capacity $C_{e}$ characterizing the energy needed to heat the whole electron subsystem, and defined as $C_{e}=\partial E / \partial T_{e}, E$ being the internal energy. At higher temperature, a very good agreement is found between $N_{e}$ evolution and previously published data [57].

\section{Electromagnetic simulations}

To estimate the effects of silicon excitation on the initiation and growth of the observed LIPSS, a careful treatment of the interaction of electromagnetic waves with rough surfaces has been performed. The FDTD method is employed to compute the inhomogeneous energy deposition below the material's rough surface. The model where Maxwell equations are coupled to free-carrier density equations for excited silicon is described in details in Ref. [70].

\section{Light propagation and material ionization}

Light propagation and ultrashort laser interaction with rough silicon surfaces are modeled by solving the system of three-dimensional Maxwell equations written in the following way:

$$
\begin{aligned}
\frac{\partial \vec{E}}{\partial t} & =\frac{\nabla \times \vec{H}}{\varepsilon_{0} \varepsilon}-\frac{1}{\varepsilon_{0} \varepsilon}\left(\vec{J}_{p}+\vec{J}_{\mathrm{Kerr}}\right) \\
\frac{\partial \vec{H}}{\partial t} & =-\frac{\nabla \times \vec{E}}{\mu_{0}},
\end{aligned}
$$

where $\vec{E}$ is the electric field, $\vec{H}$ is the magnetizing field, $\varepsilon_{0}$ is the free-space permittivity, $\mu_{0}$ is the permeability of free space, $\varepsilon=n^{2}=3.68^{2}$ is the permittivity of unexcited silicon at $800-\mathrm{nm}$ wavelength [71], $\vec{J}_{p}$ and $\vec{J}_{\text {Kerr }}$ are the nonlinear currents, which include the contribution of Kerr effect $\vec{J}_{\text {Kerr }}=$ $\varepsilon_{0} \varepsilon_{\infty} \chi_{3} \frac{\partial\left(|\vec{E}|^{2} \vec{E}\right)}{\partial t}$, where $\chi_{3}=4 \times 10^{-20} \mathrm{~m}^{2} / \mathrm{V}^{2}$ at $800 \mathrm{~nm}$ [43], and heating of the conduction band, described by the differential equation derived from the Drude model,

$$
\frac{\partial \vec{J}_{p}}{\partial t}=-v_{e} \vec{J}_{p}+\frac{e^{2} N_{e}(t)}{m_{e}^{*}} \vec{E},
$$

where $e$ is the elementary charge, $m_{e}^{*}=0.18 m_{e}$ is the reduced electron-hole mass [41], and $v_{e}=10^{15} \mathrm{~s}^{-1}$ is the electron collision frequency related to the Drude damping time [41], within the range evaluated by the time-resolved ellipsometry measurements. Above the silicon surface, the light propagation in air is calculated by Maxwell equations with $\vec{J}=0$ and $\varepsilon=1$. The system of Maxwell equations coupled with electron density equation is solved by the finite-difference numerical method [72], based on FDTD [73] and auxiliarydifferential methods for dispersive media [74]. At the edges of the grid, we apply the absorbing boundary conditions related to convolutional perfectly matched layers to avoid nonphysical reflections [75]. The initial electric field is introduced as a Gaussian slightly focused beam as follows:

$$
\begin{aligned}
E_{x}(t, r, z)= & \frac{w_{0}}{w(z)} \times \exp \left(-\frac{4 \ln 2\left(t-t_{0}\right)^{2}}{\tau_{\mathrm{FWHM}}^{2}}\right) \\
& \times \exp \left(i \omega t-\frac{r^{2}}{w(z)^{2}}-i k z-i k \frac{r^{2}}{2 R(z)}+i \varsigma(z)\right),
\end{aligned}
$$

where $\tau_{\mathrm{FWHM}}=60 \mathrm{fs}, t_{0}$ is the time delay, $w_{0}=3 \mu \mathrm{m}$ is the beam waist, $w(z)=w_{0} \sqrt{1+\left(\frac{z}{z_{R}}\right)^{2}}$ is the Gaussian's beam spot size, $\omega=2 \pi c / \lambda$ is the angular frequency, $c$ is the speed of light, $z_{R}=\frac{\pi w_{0}^{2} n_{0}}{\lambda}$ is the Rayleigh length, $r=\sqrt{x^{2}+y^{2}}$ is the radial distance from the beam waist, $R_{z}=z\left(1+\left(\frac{z_{R}}{z}\right)^{2}\right)$ is the radius of curvature of the wavelength comprising the beam, and $\varsigma(z)=\arctan \left(\frac{z}{z_{p}}\right)$ is the Gouy phase shift. The incident wave propagates along $z$ axis, the laser polarization is along $x$ axis. To account for the material ionization induced by a sufficiently intense laser field, we couple Maxwell 
equations with the kinetic equation for the electron-hole plasma as described below.

The time-dependent conduction-band carrier density evolution is described by the rate equation proposed by van Driel [76]. This equation takes into account such processes as photoionization, avalanche ionization, and Auger recombination, and is written as

$$
\frac{\partial N_{e}}{\partial t}=\frac{N_{a}-N_{e}}{N_{a}}\left(\frac{\alpha I}{\hbar \omega}+\frac{\beta I^{2}}{2 \hbar \omega}\right)+\gamma I N_{e}-\frac{C_{A} N_{e}^{3}}{C_{A} \tau_{\mathrm{rec}} N_{e}^{2}+1},
$$

where $I=\frac{n \varepsilon_{0} c}{2}|\vec{E}|^{2}$ is the intensity, $\alpha=1.02 \times 10^{5} \mathrm{~m}^{-1}$ and $\beta=1 \times 10^{-10} \mathrm{~mW}^{-1}$ are the one- and two-photon absorption coefficients, respectively $[27,42,43], N_{a}=5 \times 10^{22} \mathrm{~cm}^{-3}$ is the silicon atom density, $C_{A}=3.8 \times 10^{-31} \mathrm{~cm}^{6} / \mathrm{s}$ is the Auger recombination rate [76], $\tau_{\mathrm{rec}}=6 \times 10^{-12} \mathrm{~s}$ is the minimum Auger recombination time [77], and $\gamma=21.2 \mathrm{~cm}^{2} / \mathrm{J}$ is the avalanche ionization coefficient at $800-\mathrm{nm}$ wavelength [78].

The changes of the real and imaginary parts of the permittivity associated with the time-dependent free-carrier response can be derived from Eqs. (6) and (7) and are written as follows:

$$
\begin{aligned}
& \operatorname{Re}(\varepsilon)=\varepsilon_{\infty}-\frac{e^{2} N_{e}}{\epsilon_{0} m_{e}^{*}\left(\omega^{2}+v_{e}^{2}\right)} \\
& \operatorname{Im}(\varepsilon)=\frac{e^{2} N_{e} v_{e}}{\varepsilon_{0} m_{e}^{*} \omega\left(\omega^{2}+v_{e}^{2}\right)} .
\end{aligned}
$$

To induce light scattering, a roughness layer is introduced at the air-Si interface by a random distribution of $N$ nanocavities modeled by squared holes of characteristic length $2 l$ and depth $l$ with $n=1$ and $k=0$. Within the total considered area $S$, each nanocavity occupies an area $S_{c}$. The concentration is then defined as $C=N S_{c} / S$ and the corresponding average distance between nanocavities is $d=2 l / \sqrt{C}$.

\section{FDTD results}

Upon ultrashort laser irradiation of silicon target, the optical indices change transiently, affecting the laser propagation and influencing the resulting absorbed energy deposition. Although the transition from parallel-oriented to perpendicularoriented patterns on the silicon surface was discussed based on analytical Sipe theory and linear Maxwell equations [14,32], the nonlinear effects such as multiphoton absorption, Kerr effect, and transient generation of free carriers has not yet been considered. In fact, the nonlinearity was shown to play a defining role in the formation of HSFLs on excited fused silica surfaces [16,79]. Laser-excited silicon exhibits a rich nonlinear response in the visible spectrum range [70], therefore, the electron density kinetic evolution upon ultrashort laser irradiation is of great fundamental interest. In particularity, the optical switching of radiative optical response from a nanocavity on excited but nonmetallic silicon surface has been never reported previously. We discuss this phenomenon and relate the appearance of complex crossed-oriented patterns to this dramatic change in scattering behavior of silicon surface.

Figure 5 shows the temporal evolution of photo-excited charged carriers upon ultrashort laser irradiation of rough silicon surface. To describe the formation mechanism and the topography dynamics resulting in crossed patterns of LSFLs and HSFLs, we discuss different stages of pattern evolution, starting from the initial linear regime, corresponding to lower energy deposition. We also analyze the instantaneous absorption by a singular nanocavity for excited silicon surfaces with different free-carrier densities. At the initial stage, the freecarrier density is too low to influence the propagation of light and follows the intensity distribution. A single nanocavity on unexcited silicon exhibits a radiative optical response in the form of spherical standing waves as shown in Fig. 5(a), having more pronounced maxima along the $y$ direction than along the $x$ direction and separated by $\lambda / n \simeq 220 \mathrm{~nm}$, where $n$ is the refractive index of unexcited silicon. As a result, the subwavelength free-carrier pattern with orientation parallel to laser polarization is created on a rough surface as a coherent superposition of the waves scattered by a distribution of nanocavities and the incident wave. At a higher laser energy dose, the transient optical response is affected by the nonnegligible imaginary part of the refractive index [Fig. 5(b)]. As at the earlier stage, the maximum closer to a nanocavity is more pronounced parallel to laser polarization and the absorption is also reinforced along the $x$ direction, with the closest maximum at the near-wavelength distance. The free-carrier pattern is now characterized by a complex mixed response at the center of the laser spot and parallel-oriented features at the borders of the laser-affected region. We underline that both radiative components of the field influence the absorbed energy on the surface of nonmetallic silicon with carrier density $N_{e}<$ $2 \times 10^{21} \mathrm{~cm}^{-3}$. The interplay between these two components results in the complex crossed nanostructures for higher energy deposition [Fig. 5(c)] as they align, respectively, parallel and perpendicular to the laser polarization. The interplay here is not only related to a particular free-electron density but also results from ionization memory of the initially established patterns. The preferential orientation of the energy absorbed by a nanocavity on the surface with $N_{e}=3 \times 10^{21} \mathrm{~cm}^{-3}$ free-carrier density is now inverted. The closest maxima are more pronounced along the $x$ direction, which resembles, at first sight, the common plasmonic response on metal surfaces, albeit the silicon remains nonmetallic with $\operatorname{Re}(\tilde{n})>\operatorname{Im}(\tilde{n})$, where $\tilde{n}$ is the refractive index of excited silicon. The waves scattered from nanofeatures on non-metallic surfaces have an evanescent nature and should be referred to as quasicylindrical waves to differentiate from surface plasmon waves, requiring specific conditions and metal-dielectric interface for their excitation [25]. The crossed patterns in Fig. 5(c) occur due to extremely small differences in amplitudes of parallel and perpendicular field components. If the closest to the nanocavity standing spherical wave has a dominant contribution toward perpendicular-oriented features, the second-order spherical wave exhibits more pronounced field enhancement along the $y$ axis. Finally, as we approach the metallic state and free-electron density of $N_{e}^{\mathrm{cr}}=5 \times 10^{21} \mathrm{~cm}^{-3}$, first-order and second-order spherical waves have larger contributions along the $x$ axis. As a result, only perpendicular-oriented free-carrier density patterns are established at the center of laser spot in Fig. 5(d) with a period of around $550 \mathrm{~nm}$. The features are now mixed at the borders of the laser-affected region due to a complex electromagnetic interplay between perpendicular and parallel field contributions. To sum up, the free-carrier 

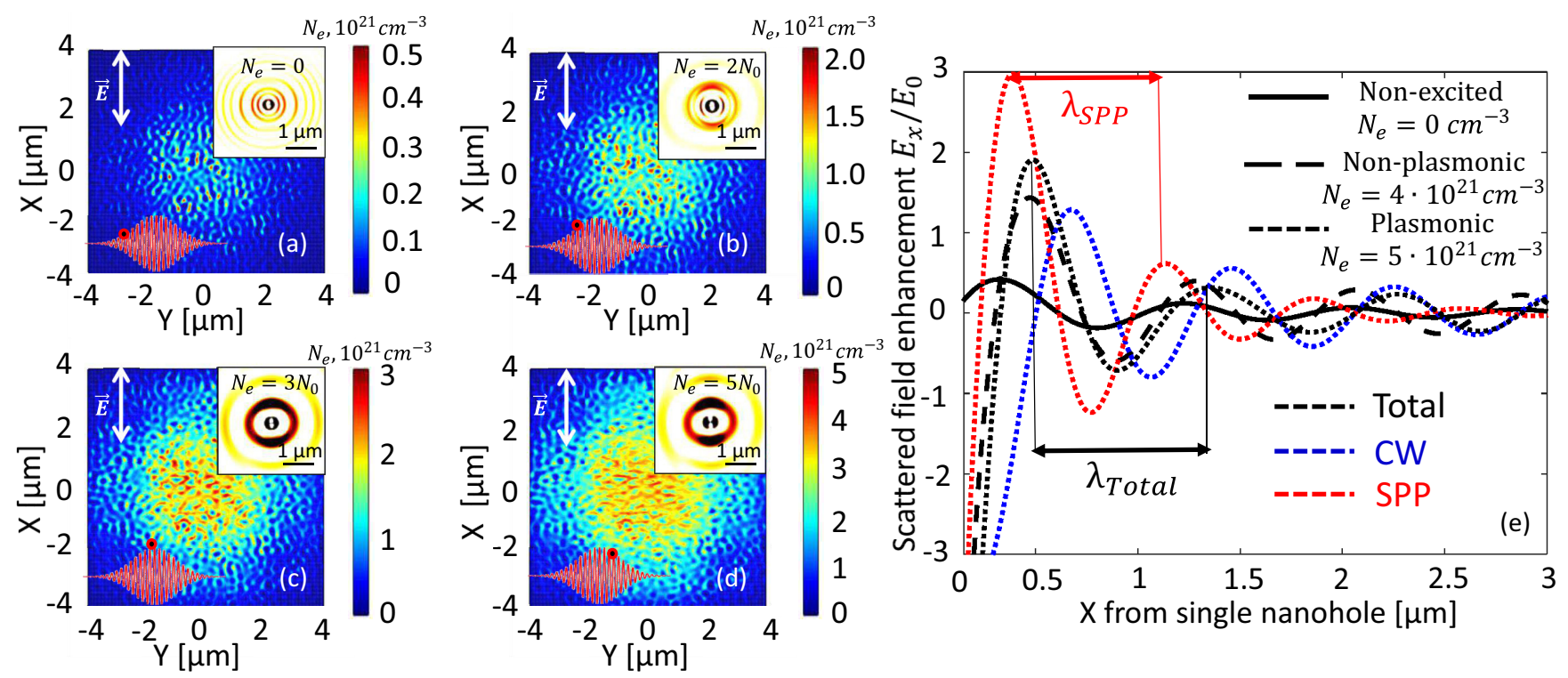

FIG. 5. (a)-(d) Free-carrier-density evolution below a rough silicon surface irradiated by an ultrashort laser pulse with a pulse duration of 60 fs and a fluence $F=0.3 \mathrm{~J} / \mathrm{cm}^{2}$. Roughness is introduced by a random distribution of nanocavities with a characteristic length $2 l=20 \mathrm{~nm}$ and a concentration $C=1 \%$. At the upper right corner of the figure, the energy absorption by a single nanocavity on the surface with homogeneous free-carrier density $N_{e}$ normalized to $N_{0}=10^{21} \mathrm{~cm}^{-3}$ is shown. At the lower left corner, the time corresponding to the current free-carrier density snapshot is indicated on the incident Gaussian pulse. (e) Electric field contributions including quasicylindrical wave (CW, blue), surface plasmon polariton (SPP, red), and total contributions (total, black) scattered by a single surface nanocavity in transverse plane for different inhomogeneous electron densities of the silicon surface. Note that SPP contributions are considered only for the highest carrier density. For nonplasmonic cases, only the total contribution is shown in the absence of surface plasmon wave.

densities of order $N_{e}=3 \times 10^{21} \mathrm{~cm}^{-3}$ for nonmetallic excited silicon induce the patterns with crossed perpendicular and parallel orientations to laser polarization.

The obtained electron density patterns, however, can hardly explain the formation of LIPSSs observed experimentally. First, for low-excitation conditions, no permanent modification is expected, as the temperatures are below the melting point, which is also supported by the fact that the HSFL parallel to laser polarization alone without LSFLs for similar excitation conditions are rarely observed experimentally [80]. Second, the patterns in Fig. 5(c) present no clear structure of ripples with well-defined regions, whereas perpendicularoriented patterns occupy the center of the modified region in Fig. 5(d), which contradicts the experimental results, where parallel-oriented HSFLs are in the center, surrounded by perpendicularly oriented LSFLs (see Fig. 6 for example). Finally, if we assume that both kinds of LIPSSs have similar electromagnetic origin, then the central region of modification should correspond to smaller free-carrier electron densities, which is not possible due to Gaussian energy deposition of the laser pulse. Therefore, current FDTD results taking account for transient optical response, in good agreement with previous FDTD calculations for fixed optical properties [14,32], can only explain the formation of classical LSFLs perpendicular to laser polarization on laser-excited silicon surfaces. The origin of HSFLs parallel to laser polarization is further investigated in the Discussion.

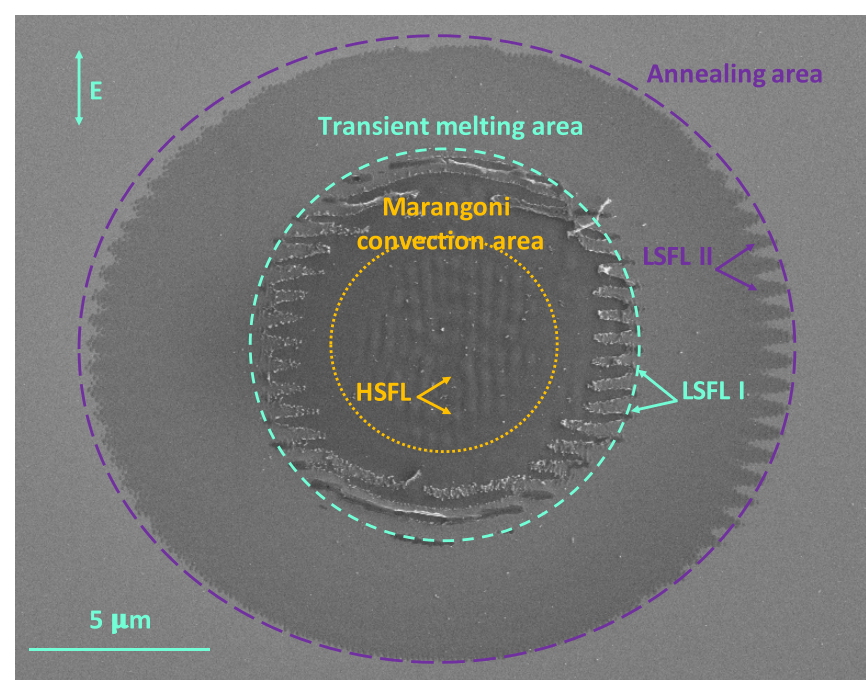

FIG. 6. A site on silicon irradiated by 30 laser pulses of $60 \mathrm{fs}$ at $0.22 \mathrm{~J} / \mathrm{cm}^{2}$. Three areas are identified with a threshold marked by a circle. The larger zone encompasses the annealing process area, the intermediate zone covers the transient melting area, and the central zone undergoes significant capillary gradients. Scanning electron microscopy (SEM) image reveals HSFL with $\Lambda_{\mathrm{HSFL}}=400 \mathrm{~nm}$ at the center of the impact and LSFL with $\Lambda_{\mathrm{LSFL}}=730 \mathrm{~nm}$, referred to as LSFL I in the melting area and LSFL II in the annular annealing area. The laser polarization is indicated by the arrow in the upper-left corner. 


\section{Role of CW and SPP in LSFL formation}

The formation of perpendicular-oriented near-wavelength patterns for much weaker excitation conditions than required for surface plasmon wave excitation $[\operatorname{Re}(\varepsilon)<-1]$ was reported in several works based on the absorption energy distributions below the laser-excited surface calculated by full-vector Maxwell equations [14,16,32]. This result is also consistent with the presence of a scattered field solution on rough surfaces responsible for energy modulation perpendicular to laser polarization, different from SPP, present in the macroscopic Sipe model [22,31]. Recently, this solution has been attributed to quasicylindrical evanescent wave (CW) [25], excited microscopically by a single surface dipole (for example, a nanocavity) and playing an important role in both nonplasmonic and plasmonic materials [81,82]. The complex nature of the absorbed energy modulation indicates that several statements based solely on the presence of surface plasmon wave should be revisited. For instance, the surface plasmon wave solution is proportional to $\exp ($ iqx $)$, where $q=\frac{2 \pi}{\lambda_{\mathrm{SPP}}}=\frac{2 \pi}{\lambda} \sqrt{\frac{\varepsilon}{\varepsilon+1}}$ is the wave vector defining the surface plasmon wavelength $\lambda_{\mathrm{SPP}}$. This wavelength changes upon laser excitation due to the transient optical properties of silicon, resulting in $700-800 \mathrm{~nm}$ periods for excitation laser wavelength $\lambda=800 \mathrm{~nm}$ in the range, where conditions for surface plasmon excitation are satisfied [27,31]. This periodic modulation was proposed as an explanation to LIPSS periods, smaller than laser wavelength. However, the presence of quasi-cylindrical wave having a space-shifted modulation with $\lambda_{\mathrm{CW}}$, coinciding with the laser wavelength, influences the resulting patterns. The SPP and CW contributions are separated from the total field scattered by a single nanocavity for different surface excitation conditions in Fig. 5(e), following the analytical approach described in Ref. [83]. For nonplasmonic cases, only total contribution is shown as it coincides with the $\mathrm{CW}$. The black solid line indicates that the periodic modulation perpendicular to laser polarization is still present for unexcited silicon [as well as in the absorbed energy distribution from a single nanocavity in FDTD simulations shown in Fig. 5(a)]. However, the intensity maxima along $x$ appear to be significantly smaller than along $y$. For nonplasmonic excitation conditions $N_{e}=4 \times 10^{21} \mathrm{~cm}^{-3}$ (black dashed line), the contribution of CW increases. For such conditions, the intensity maxima along $x$ prevail over the ones along $y$. For plasmonic excitation conditions $N_{e}=$ $5 \times 10^{21} \mathrm{~cm}^{-3}$ (black dotted line), both $\mathrm{CW}$ with $\lambda_{\mathrm{CW}} \simeq 800$ $\mathrm{nm}$ and SPP with $\lambda_{\mathrm{SPP}} \simeq 720 \mathrm{~nm}$ solutions influence the result. The total periodic modulation $\lambda_{\text {total }}=810-830 \mathrm{~nm}$ deviates from both SPP and CW and changes with distance from the nanocavity. The position of maxima from the analytical solution agree well with the absorbed energy maxima calculated by FDTD from a single nanocavity and shown in Fig. 5(d). The first pronounced maximum belongs to SPP, however, the solution decays faster than CW. At distances $>2 \mu \mathrm{m}$, the total contribution almost coincides with $\mathrm{CW}$. Such behavior is similar to lossy metals and, for example, long excitation wavelengths, where the Norton wave, which is a part of the $\mathrm{CW}$ at the long-distance limit, dominates over SPP [82]. Interestingly, there is no pronounced difference between the maximum field enhancements related to nonplasmonic and plasmonic cases for silicon. The presence of both $\mathrm{CW}$ and SPP solutions makes it difficult to have a resulting periodic modulation with a wavelength significantly different from the laser excitation wavelength. A different scenario to explain periods much smaller than the laser wavelength was proposed in Ref. [25]. It was shown that the first total electric field maximum plays a defining role, therefore, the distance between a single nanovity and this field maximum would define the structure and the dynamics of periodic patterns in multipulse laser irradiation. For both nonplasmonic and plasmonic excitation conditions for silicon $N_{e}=4 \times 10^{21} \mathrm{~cm}^{-3}$ and $N_{e}=5 \times 10^{21} \mathrm{~cm}^{-3}$, this distance corresponds to around $500 \mathrm{~nm}$ and defines the minimum possible distance between neighboring nanostructures. The multipulse evolution, however, is more complicated due to inhomogeneous distribution of free carriers in ripple crests and troughs once the ripples are formed-enhanced transient absorption and stronger excitation with the increasing number of pulses due to nanostructure development and growth. For example, it was shown that for plasmonic metals with less pronounced losses and comparable contributions of SPP with $\lambda_{\mathrm{SPP}} \simeq \lambda$ and $\mathrm{CW}$, the distance between a single hole and the first intensity enhancement occurs close to $3 \lambda / 4=600 \mathrm{~nm}$.

\section{DISCUSSION}

During repeated photoexcitation as shown in Fig. 1, the electron density is inhomogeneously distributed over the surface due to the Gaussian distribution of the beam and the near-field effects associated with transiently formed nanoreliefs. Multipulse dynamics of surface topography is driven by the interplay of electromagnetic and hydrodynamic processes, as the energy absorption below the surface cavities and ripple hollows results in a higher absorption. Throughout this profile deepening and roughness amplification mechanism by positive feedback, LIPSSs once formed are not erased but only become more pronounced by electromagnetic effects and pulse-by-pulse material movement. In spite of the selforganization complexity, the generation of LIPSSs on silicon requires a precise value of the electron density in the conduction band that could be viewed as a microscopic emergence criterion. Emergence is a key concept in self-organization and variabilities as periodicity decreases and domain bifurcation increases with the number of pulses $N$ are more related to the natural evolution of the system under organization. Therefore, ellipsometric pump-probe measurements, ab initio, and electromagnetic calculations agree toward an emergence value of $N_{e}^{\mathrm{em}}=3 \times 10^{21} \mathrm{~cm}^{-3}$ to achieve the desired structures. This value corresponds to the maximum density expected in the periodically spaced intensity maxima corresponding to the location of constructive interference of a surface wave with the incident wave. The local absorbed electromagnetic energy induces a strong location-dependent electron temperature and, after relaxation with the lattice, spatially heterogeneous heating, and phase transitions. Thus, after $N$ pulses, the energy distribution is assumed to convolute the distribution of the resulting interference field with the hydrodynamic response of the material. This complexity associated with the difficulty of probing locally, with a necessary spatial resolution below $100 \mathrm{~nm}$ and an intrapulse temporal resolution of the order of 
$10 \mathrm{fs}$, justifies the exploitation of postmortem cross patterns to derive a coherent scenario of LIPSS formation on the basis of the measurements and calculations presented in the previous sections.

Let us start by discussing the LSFL structures of $\Lambda \simeq$ $760 \mathrm{~nm}$ period observed in Fig. 1. This value of $\Lambda$ is very close to the laser wavelength, as expected for a relatively small number of pulses $[25,84]$. The lower values are not necessarily related to a higher concentration of carriers modifying the surface plasmon period. As observed for metals, the period decay with $N$ is related to a strengthening of the dipole coupling for small spacings between transient nanostructures, with a typical saturation of about $3 \lambda / 4$ [25]. Also, these mechanisms put forward an ablation process localized at the maximum absorbed intensity, generating a local crater with a contrast of up to $300 \mathrm{~nm}$ with respect to the adjacent surface. As shown by the DFT, to support a stationary value of $N_{e}^{\mathrm{em}}$, a thermalization of the carriers at a temperature of $T_{e}^{\mathrm{em}}=8000 \mathrm{~K}$ is required, allowing us to estimate the associated electron energy $\Delta E_{\mathrm{em}}=\int_{T_{e}^{0}}^{T_{e}^{\mathrm{em}}} C_{e} d T_{e} \simeq 2.5 \times 10^{9} \mathrm{~J} / \mathrm{m}^{3}$, and by extrapolation of the final local heating of the lattice $\Delta T_{i}$ after relaxation. It is important to note that for $N_{e}^{\mathrm{em}}$ the energy absorption length is about $\delta_{\mathrm{abs}}=94 \mathrm{~nm}$ and that it remains more important than the thermal diffusion length estimated at about $\delta_{\text {diff }}=20 \mathrm{~nm}$ for $T_{e}=8000 \mathrm{~K}$ in silicon. Thus, as a first estimation, we can estimate the local heating of the lattice without any electron transport and $\Delta T_{i}=\Delta E_{\mathrm{em}} / C_{i}^{s} \simeq 1250 \mathrm{~K}$ with $C_{i}^{s} \simeq 2 \times 10^{6} \mathrm{~J} / \mathrm{K}$, suggesting a lattice heating is ineffective to reach the crystal melting transition at $T_{m}=1687 \mathrm{~K}$ as well as to exceed the latent heat of the crystalline-liquid transition $\Delta H_{\text {fus }}^{\mathrm{cl}} \simeq 4.2 \times 10^{9} \mathrm{~J} / \mathrm{m}^{3}$. This conclusion still holds for the highest electron density of $N_{e}^{\mathrm{em}}=5 \times 10^{21} \mathrm{~cm}^{-3}$, allowing only heating up to the required temperature for melting. For sufficiently strong ultrafast laser pulse excitation, interatomic bonds can be softened on a subpicosecond timescale less than a phonon period, referred to as ultrafast melting concept [85]. The lattice atoms start to move away from their equilibrium positions, collectively in the same direction and up to the bond breaks. This mechanism has already been invoked to explain the phase transition in the context of LIPSS formation [86]. However, $a b$ initio calculations performed on photoexcited silicon, in full agreement with the DFT calculation results reported in this paper show that ultrafast melting requires a relatively high criterion for electron-hole pair excitation. More than $9 \%$ of the valence electrons, corresponding to $N_{e}=18 \times$ $10^{21} \mathrm{~cm}^{-3}$ are supposed to be in the conduction band to induce an atomic motion overcoming the Lindemann criterium of stability [57,87]. The Fermi-Dirac distribution fulfilling this condition corresponds to an electron temperature larger than $17000 \mathrm{~K}$ [87], thus twice the value of $T_{e}=8000 \mathrm{~K}$ we have estimated from our experiments. We also emphasize that the energy required for ultrafast melting is on the same order of magnitude as the normal melting energy.

Contrary to what is commonly known about metals, the results therefore suggest that LSFL structures are obtained under energy conditions insufficient to allow the formation of a liquid layer that would be the site of periodic and localized ablation in the zones of highest intensity [88]. This submelting condition has already been put forward by other authors for silicon $[20,89]$. However, the presence of an amorphous phase state observed by TEM can raise questions if a liquid layer is not formed. It is generally accepted that local temperature gradients could affect solidification speed and the transient cooling conditions after melting, determining the final structural phase [90,91]. In particular, a molten zone can solidify so rapidly that crystallization is bypassed. A process of lattice softening of heated silicon undercooled at a rate too fast for the nucleation of $c$-Si favoring $a$-Si formation has also been suggested [92]. As discussed below, we propose that at high quenching rates such as those experienced by the material being irradiated by an ultrashort laser pulse, on the order of $10^{12} \mathrm{Ks}^{-1}$, amorphization can proceed at temperatures well below the standard melting point.

The polarization-dependent process of LSFL formation is initiated by inhomogeneous energy absorption around random nanoreliefs where the electromagnetic patterns show local field enhancement and far-field interference with scattered wave. For metals, after the first laser shots, a destabilization of the heated layer occurs mainly due to the formation of a rarefaction wave and potential cavitation process below the surface. An order is progressively emerging as hot laser spots driven by a nonradiative response of nanoscale perturbations superimpose with the coherent radiative response. For silicon, cross-section imaging shown in Fig. 1(e) does not show mechanically generated nanocavities or nanovoids as observed for metals [93]. Transient laser-induced surface nanorecessions are not readily formed even if annular region seen in Fig. 1 exhibits a clear nanoscale topography modification after 13 pulses [Fig. 1(a)]. However, the maxima of intensity trigger a localized heating throughout the electronphonon energy transfer and are associated with a pressure increase of several $\mathrm{GPa}$ in a periodically modulated manner [21]. Upon this specific thermodynamic conditions [94], the softening state of photoexcited silicon likely turns to a melting state several hundreds of Kelvin below the standard $T_{m}$ value. Within consecutive tens of picoseconds, the pressure is released as the rarefaction wave follows the shock wave due to longitudinal temperature gradients. The stretched liquid state undergoes an amorphization process rather than cavitation if the tensile limit of about $-3 \mathrm{GPa}$ is not reached. A phase change between liquid phase and amorphous solid has been shown to appear near $1450 \mathrm{~K}[95,96]$. Upon successive ultrafast heating by laser pulse, the amorphous area is expected to grow as the material may melt via a first-order transition at a temperature up to $300 \mathrm{~K}$ below $T_{m}$ with a reduced latent heat as $\Delta H_{\text {fus }}^{a l} \simeq 0.3 \Delta H_{\text {fus }}^{c l}$ [97]. The optical properties of $a$-Si at $800 \mathrm{~nm}(\varepsilon=15.167+i 0.85)$ do not differ much from those of $c$-Si to significantly alter the role of the optical response during the feedback [98].

The formation of HSFLs is observed in Fig. 1 inside an annular region where they overlap with LSFLs, producing a crossed-pattern of LIPSSs. More precisely, HSFLs grow between neighbor LSFL crests and could benefit from a higher energy confinement as the field is strengthened in valleys and nanocavities. The heating conditions are therefore at least as high for LSFLs or even higher due to local field enhancement. One can even wonder if their presence is conditioned or not by the presence of LSFLs, as they do not appear at the edge of the impact. For slightly lower excitation conditions but a higher number of pulses, a different LIPSS 
arrangement is exhibited in Fig. 6. HSFLs are formed in the center of the affected region, whereas LSFLs are found at the edges. Note that the FDTD simulations reveal that the contribution of the electric field into electron density patterns perpendicular to laser polarization evidenced in Figs. 5(d) and 5(e) is significant even for excitation conditions well below the damage threshold and LSFL pattern establishment. Such pronounced electronic patterns create significant temperature gradients $\Delta T_{i}=100 \mathrm{~K} / \mathrm{nm}$ on silicon surfaces, which can directly render the system unstable, producing hydrothermal waves perpendicular to produced temperature gradients and resulting parallel to initially set laser polarization. Driven by surface tension-driven Marangoni forces, inhomogeneously heated melt flow moves the material from hot spots to colder zones and eventually cools down by thermal diffusion. The soft matter or melt front stops, freezing nanostructures in an amorphous phase upon lattice cooling. The direction of HSFL alignment is governed by the polarization-dependent temperature gradients and the observed periodicity can be derived from the thermocapillary-driven convection length related to Marangoni instability as $\lambda_{\mathrm{Ma}}=2 \pi h / \sqrt{\mathrm{Ma} / 8}$ where $\mathrm{Ma}=$ $\frac{\gamma \Delta T_{i} h}{\mu D_{i}}$ is the Marangoni number and $D_{i}=k_{i} / C_{i}$ is the thermal diffusivity. The undetermined melt-layer thickness is crucial in this estimation and supposing a range of $h=20-50 \mathrm{~nm}$ as the thickness of the amorphous layer between the LSFL crests, a spectrum of $\lambda_{\mathrm{Ma}}=280-450 \mathrm{~nm}$ can be predicted using reported values of liquid silicon data $[99,100]$. These periods are in good agreement with $\Lambda_{\mathrm{HSFL}}=330 \mathrm{~nm}$ provided by AFM measurements. Finally, under Marangoni forces, the dynamics of fluid flows is expected to arrange into regular nanopatterns inheriting the light polarization response on roughness with a periodicity stabilized at a critical wavelength depending on the melt-layer thickness. Moreover, hydrothermal waves, driving the hotter melt flow above the initial surface level, were shown to be at the origin of HSFL growth in similar irradiation conditions for metals [21]. Extrapolating these simulation results for $\mathrm{Si}$, the related characteristic times of fluid flow due to surface tension difference between LIPSS hollows is estimated as $\tau_{T}=\frac{\mu L^{2}}{4 h \gamma \Delta T}$ where $\mu=0.874 \times 10^{-3} \mathrm{~Pa} . \mathrm{s}$ is the silicon viscosity around melting temperature, $L=\Lambda_{\mathrm{HSFL}}=$ $330 \mathrm{~nm}$ and $h \simeq 50 \mathrm{~nm}, \gamma=0.8 \times 10^{-3} \mathrm{~N} / \mathrm{m} / \mathrm{K}$ [101]. We note that the energy is efficiently deposited below the LSFL hollows where the electron plasma density might trigger significantly larger temperatures than between the ridges due to local field enhancement. A factor of 2 in the absorbed energy is revealed by FDTD simulations that potentially leads to at least $\Delta T=10^{3} \mathrm{~K}$ difference close to the melting threshold. Based on these estimations, $\tau_{T} \simeq 500 \mathrm{ps}$ is obtained in accordance with the expected liquid lifetime [102,103]. In addition, the process may be accelerated by pressure differences due to the shock wave release. In that respect, a typical pressure gradient of $\Delta P=1 \mathrm{GPa}$ would drive up a similar displacement during a typical time $\tau_{P} \simeq \frac{\mu L^{2}}{4 h^{2} \Delta P} \simeq$ $9 \mathrm{ps}$, which is also within the molten-layer lifetime. As a result, in the transverse plane, material density patterns forming HSFLs are proposed to be established upon fluid flow driven by transverse Marangoni gradients and strengthened by local pressure gradients. The self-organization mechanism is also amplified through positive feedback as reversible transports of matter governed by hydrodynamics are combined to irreversible light scattering processes, leading to dissipative structures formation [104,105].

To validate the proposed scenario for both HSFL and LSFL formation on silicon, a laser impact has been studied at a peak fluence $25 \%$ lower that shifts the crossed-pattern features toward the center of the impact. A higher number of pulses $N=30$ was needed to present the two kinds of nanostructures. The result revealed by SEM is presented in Fig. 6. For $F=0.22 \mathrm{~J} / \mathrm{cm}^{2}$, HSFLs are formed at the center of the impact whereas LSFLs are visible in an outer region on the border of two areas marked by different contrasts. To simplify the discussion, we have identified three zones that are encircled in Fig. 6. The largest radius is supposed to be representative of the zone whose microstructure has been altered and corresponds to the annealing process as previously proposed [90]. At the edge of this zone, some fingers are clearly visible, referred to as LSFL II, and show that the energy deposition is modulated even if the material does not undergo a phase transition. This kind of coherent LIPSS is directly subsequent to the electromagnetic response impressed on the surface. The intermediate circle represents a region heated at highest temperature, implying undoubtedly a final structural state different from the pristine one. The material has probably reached the discussed melting threshold and information obtained from Fig. 1 suggests that the high quenching rate has favored an undercooling process and an amorphous state. Here, LSFL I may consist of a periodic arrangement of structural states. A similar period is measured for LSFLs I and II, supporting that they result from the same electromagnetic process even if thermodynamic conditions differ. Finally, the heart of the impact consists of a thin lasermelted layer that can be destabilized by local temperature gradients. As for the higher fluence case, the nonradiative contribution of the light inhomogeneous absorption triggers a convection instability. This activates HSFL formation parallel to the laser polarization with a period slightly larger than previously observed. LSFLs are not observed jointly to HSFLs but their remnants at a lower fluence in the Gaussian distribution strongly suggest that they were transiently formed at the center but faded by the HSFL generation. Also, for $N=15$ laser shots (not shown here), LSFLs cover the impact and HSFLs were not observed. This second irradiation condition with an enhanced feedback finally presents the interest of decoupling the two types of structures, showing that a LSFL is the direct result of a primary process whereas HSFL formation requires a well-defined photoexcitation and restrictive thermomechanical conditions.

As a result, upon ultrafast laser excitation, a modulated energy deposition driven by light scattering is supposed to heat locally the surface at a temperature below $T_{m}$ at standard conditions. However, the isochoric heating induces high positive pressure, allowing the local melting of the material. The liquid survive a few tens of picoseconds under strong undercooling conditions and the rarefaction wave combined with rapid cooling conditions favor a convection instability forming nanoplots of amorphous state. The sequence of compressive and tensile stresses under nonhomogeneous melting irradiation conditions suggest favoring a periodic amorphization process rather than cavitation, in contrast to what is 
observed for higher fluence conditions on silicon or metals. The concomitant formation of LSFLs and HSFLs can be controlled by a fine energy tuning around the melting threshold, increasing the pulse number and thus the positive feedback mechanisms.

\section{CONCLUSIONS}

This paper sheds light on the intricate role of photoexcitation, inhomogeneous absorption, and hydrodynamic mechanisms driving periodic phase transitions on ultrafast laser-irradiated silicon surfaces. Upon multiple pulse irradiation of silicon, crossed patterns of nanostructures were generated by a superposition of HSFLs perpendicular to LSFLs. Relatively precise values of the photoexcitation and relaxation dynamics of the free-electron population were deduced from time-resolved experiments. For LIPSS-formation conditions, a generated free electron density of around (3 \pm 2) $\times 10^{21} \mathrm{~cm}^{-3}$ was estimated from time-resolved ellipsometry and supported by TDDFT calculations. Charge density in the conduction band rapidly thermalizes, determining the degree of heating up to $8000 \mathrm{~K}$ in the free-electron population.

Spatially distributed ultrafast transitions, composed of $a$ $\mathrm{Si}$, originate from excited electrons energy transfer associated with strong pressure gradients. Upon multiple pulse irradiation, morphology-mediated feedback effects strengthen the energy deposition where the electromagnetic response is enhanced. In the chosen conditions of excitation, a dual nature composed of a coherent superposition of the incident light implying a quasicylindrical scattered wave rather than surface plasmon and local field enhancement around surface heterogeneities fosters thermomechanical conditions driving the local melting and amorphization process in a periodic manner. Driven by nonradiative fields, HSFL formation requires the formation of a thin liquid layer destabilized by high-temperature gradients involving a Marangoni convection process. The periodic emergence of the amorphous phase is finally attributed to the successive cycles of polarizationdependent light organization around progressively formed surface nanoreliefs undergoing compression and rarefaction sequence. This work paves the way toward the ordering of periodic structural modifications beyond the diffraction limit via the control of spatial light confinement on the surface.

\section{ACKNOWLEDGMENTS}

This work was supported by the ANR project DYLIPSS (ANR-12-IS04-0002-01) and by the LABEX MANUTECHSISE (ANR-10-LABX-0075) of the Université de Lyon, within the program Investissements d'Avenir (ANR-11IDEX-0007) operated by the French National Research Agency (ANR). Numerical calculations have been performed using HPC resources from GENCI-TGCC and CINES, project gen7041. This work was also supported by the IMOTEP project within the program Investissements d'Avenir operated by ADEME.
[1] K. Sugioka and Y. Cheng, Light: Sci. Appl. 3, e149 (2014).

[2] M. Domke, B. Egle, G. Piredda, S. Stroj, G. Fasching, M. Bodea, and E. Schwarz, J. Micromech. Microeng. 26, 115004 (2016).

[3] J. Bonse, S. Höhm, S. V. Kirner, A. Rosenfeld, and J. Krüger, IEEE J. Sel. Top. Quantum Electron. 23, 1 (2017).

[4] T. Baldacchini, J. E. Carey, M. Zhou, and E. Mazur, Langmuir 22, 4917 (2006).

[5] B. Dusser, Z. Sagan, H. Soder, N. Faure, J.-P. Colombier, M. Jourlin, and E. Audouard, Opt. Express 18, 2913 (2010).

[6] T.-H. Her, R. J. Finlay, C. Wu, S. Deliwala, and E. Mazur, Appl. Phys. Lett. 73, 1673 (1998).

[7] M. Halbwax, T. Sarnet, P. Delaporte, M. Sentis, H. Etienne, F. Torregrosa, V. Vervisch, I. Perichaud, and S. Martinuzzi, Thin Solid Films 516, 6791 (2008).

[8] D. Scorticati, G.-W. Römer, D. F. de Lange, and B. Huis in 't Veld, J. Nanophotonics 6, 063528 (2012).

[9] A. Y. Vorobyev, V. S. Makin, and C. Guo, Phys. Rev. Lett. 102, 234301 (2009)

[10] A. Papadopoulos, E. Skoulas, A. Mimidis, G. Perrakis, G. Kenanakis, G. D. Tsibidis, and E. Stratakis, Adv. Mater. 31, 1901123 (2019).

[11] N. Tagawa, M. Takada, A. Mori, H. Sawada, and K. Kawahara, Tribol. Lett. 24, 143 (2006).

[12] E. Rebollar, I. Frischauf, M. Olbrich, T. Peterbauer, S. Hering, J. Preiner, P. Hinterdorfer, C. Romanin, and J. Heitz, Biomaterials 29, 1796 (2008).
[13] J. Z. P. Skolski, G. R. B. E. Romer, J. Vincenc Oboňa, and A. J. Huis in't Veld, J. Appl. Phys. 115, 103102 (2014).

[14] H. Zhang, J.-P. Colombier, C. Li, N. Faure, G. Cheng, and R. Stoian, Phys. Rev. B 92, 174109 (2015).

[15] C. Li, G.-H. Cheng, J.-P. Colombier, N. Faure, S. Reynaud, H. Zhang, D. Jamon, and R. Stoian, J. Opt. 18, 015402 (2015).

[16] A. Rudenko, J.-P. Colombier, S. Höhm, A. Rosenfeld, J. Krüger, J. Bonse, and T. E. Itina, Sci. Rep. 7, 12306 (2017).

[17] A. V. Dostovalov, T. J.-Y. Derrien, S. A. Lizunov, F. Přeučil, K. A. Okotrub, T. Mocek, V. P. Korolkov, S. A. Babin, and N. M. Bulgakova, Appl. Surf. Sci. 491, 650 (2019).

[18] C. Florian, J.-L. Déziel, S. V. Kirner, J. Siegel, and J. Bonse, Nanomaterials 10, 147 (2020).

[19] J. Reif, O. Varlamova, S. Uhlig, S. Varlamov, and M. Bestehorn, Appl. Phys. A 117, 179 (2014).

[20] G. D. Tsibidis, M. Barberoglou, P. A. Loukakos, E. Stratakis, and C. Fotakis, Phys. Rev. B 86, 115316 (2012).

[21] A. Rudenko, A. Abou-Saleh, F. Pigeon, C. Mauclair, F. Garrelie, R. Stoian, and J.-P. Colombier, Acta Mater. 194, 93 (2020).

[22] J. E. Sipe, J. F. Young, J. S. Preston, and H. M. Van Driel, Phys. Rev. B 27, 1141 (1983).

[23] M. Huang, F. Zhao, Y. Cheng, N. Xu, and Z. Xu, Acs Nano 3, 4062 (2009).

[24] F. Garrelie, J.-P. Colombier, F. Pigeon, S. Tonchev, N. Faure, M. Bounhalli, S. Reynaud, and O. Parriaux, Opt. Express 19, 9035 (2011). 
[25] A. Rudenko, C. Mauclair, F. Garrelie, R. Stoian, and J.-P. Colombier, Nanophotonics 8, 459 (2019).

[26] J. Bonse, J. Krüger, S. Höhm, and A. Rosenfeld, J. Laser Appl. 24, 042006 (2012).

[27] T. J.-Y. Derrien, T. E. Itina, R. Torres, T. Sarnet, and M. Sentis, J. Appl. Phys. 114, 083104 (2013).

[28] E. Petrakakis, G. D. Tsibidis, and E. Stratakis, Phys. Rev. B 99, 195201 (2019).

[29] A. Rudenko, C. Mauclair, F. Garrelie, R. Stoian, and J.-P. Colombier, Appl. Surf. Sci. 470, 228 (2019).

[30] A. Rämer, O. Osmani, and B. Rethfeld, J. Appl. Phys. 116, 053508 (2014).

[31] J. Bonse, A. Rosenfeld, and J. Krüger, J. Appl. Phys. 106, 104910 (2009).

[32] J. Z. P. Skolski, G. R. B. E. Römer, J. V. Obona, V. Ocelik, A. J. Huis in 't Veld, and J. T. M. De Hosson, Phys. Rev. B 85, 075320 (2012).

[33] G. R. B. E. Römer, J. Z. P. Skolski, J. Vincenc Oboňa, and A. J. Huis in 't Veld, Phys. Procedia 56, 1325 (2014).

[34] J. Bonse, J. Wrobel, J. Krüger, and W. Kautek, Appl. Phys. A 72, 89 (2001).

[35] V. Ocelík, J. V. Oboňa, J. Z. P. Skolski, G.-W. R. B. E. Römer, A. J. Huis in t'Veld, and Jeff Th. M. De Hosson, J. Laser Applications 2014, 141 (2014).

[36] A. Borowiec, M. Mackenzie, G. Weatherly, and H. Haugen, Appl. Phys. A 76, 201 (2003).

[37] A. Cullis, N. Chew, H. Webber, and D. J. Smith, J. Cryst. Growth 68, 624 (1984).

[38] H. Zhang, J.-P. Colombier, and S. Witte, Phys. Rev. B 101, 245430 (2020).

[39] T. Maitland and S. Sitzman, Electron backscatter diffraction (EBSD) technique and material characterization examples, in Scanning Microscopy for Nanotechnology, edited by W. Zhou and Z. L. Wang (Springer, New York, 2007), pp. 41-75.

[40] Y. Fuentes-Edfuf, M. Garcia-Lechuga, D. Puerto, C. Florian, A. Garcia-Leis, S. Sanchez-Cortes, J. Solis, and J. Siegel, Sci. Rep. 7, 4594 (2017).

[41] K. Sokolowski-Tinten and D. von der Linde, Phys. Rev. B 61, 2643 (2000).

[42] T. Y. Choi and C. P. Grigoropoulos, J. Appl. Phys. 92, 4918 (2002).

[43] A. D. Bristow, N. Rotenberg, and H. M. Van Driel, Appl. Phys. Lett. 90, 191104 (2007).

[44] E. D. Palik, Handbook of Optical Constants of Solids (Academic Press, San Diego, 1998), Vol. 3.

[45] C. Roeser, A.-T. Kim, J. Callan, L. Huang, E. Glezer, Y. Siegal, and E. Mazur, Rev. Sci. Instrum. 74, 3413 (2003).

[46] E. Bévillon, J.-P. Colombier, V. Recoules, H. Zhang, C. Li, and R. Stoian, Phys. Rev. B 93, 165416 (2016).

[47] H. Zhang, C. Li, E. Bévillon, G. Cheng, J.-P. Colombier, and R. Stoian, Phys. Rev. B 94, 224103 (2016).

[48] K. Yabana, T. Sugiyama, Y. Shinohara, T. Otobe, and G. F. Bertsch, Phys. Rev. B 85, 045134 (2012).

[49] D. Yu, L. Jiang, F. Wang, L. Qu, and Y. Lu, Appl. Phys. A 122, 494 (2016).

[50] X. Andrade, J. Alberdi-Rodriguez, D. A. Strubbe, M. J. T. Oliveira, Fernando Nogueira, A. Castro, J. Muguerza, A. Arruabarrena, S. G. Louie, A. Aspuru-Guzik, Angel Rubio, and M. A. L. Marques, J. Phys.: Condens. Matter 24, 233202 (2012).
[51] F. Tran and P. Blaha, Phys. Rev. Lett. 102, 226401 (2009).

[52] S. A. Sato, Y. Taniguchi, Y. Shinohara, and K. Yabana, J. Chem. Phys. 143, 224116 (2015).

[53] N. Troullier and J. L. Martins, Phys. Rev. B 43, 1993 (1991).

[54] T. Otobe, M. Yamagiwa, J.-I. Iwata, K. Yabana, T. Nakatsukasa, and G. F. Bertsch, Phys. Rev. B 77, 165104 (2008).

[55] E. Yablonovitch, J. P. Heritage, D. E. Aspnes, and Y. Yafet, Phys. Rev. Lett. 63, 976 (1989).

[56] A. Sommer, E. M. Bothschafter, S. A. Sato, C. Jakubeit, T. Latka, O. Razskazovskaya, H. Fattahi, M. Jobst, W. Schweinberger, V. Shirvanyan, V. S. Yakovlev, R. Kienberger, K. Yabana, N. Karpowicz, M. Schultze, and F. Krausz, Nature 534, 86 (2016).

[57] E. S. Zijlstra, A. Kalitsov, T. Zier, and M. E. Garcia, Adv. Mater. 25, 5605 (2013).

[58] J. F. Young and H. M. van Driel, Phys. Rev. B 26, 2147 (1982).

[59] X. Gonze, B. Amadon, P.-M. Anglade, J.-M. Beuken, F. Bottin, P. Boulanger, F. Bruneval, D. Caliste, R. Caracas, M. Cote et al., Comput. Phys. Commun. 180, 2582 (2009).

[60] P. Hohenberg and W. Kohn, Phys. Rev. 136, B864 (1964).

[61] W. Kohn and L. J. Sham, Phys. Rev. 140, A1133 (1965).

[62] N. D. Mermin, Phys. Rev. 137, A1441 (1965).

[63] J. P. Perdew, K. Burke, and M. Ernzerhof, Phys. Rev. Lett. 77, 3865 (1996)

[64] M. Torrent, F. Jollet, F. Bottin, G. Zérah, and X. Gonze, Comput. Mater. Sci. 42, 337 (2008).

[65] H. J. Monkhorst and J. D. Pack, Phys. Rev. B 13, 5188 (1976).

[66] C. Kittel, Introduction to Solid State Physics, 8th ed. (John Wiley \& Sons, New York, 2004).

[67] R. W. Godby, M. Schlüter, and L. J. Sham, Phys. Rev. Lett. 56, 2415 (1986).

[68] V. Recoules, J. Clérouin, G. Zérah, P. M. Anglade, and S. Mazevet, Phys. Rev. Lett. 96, 055503 (2006).

[69] E. Bévillon, J.-P. Colombier, V. Recoules, and R. Stoian, Phys. Rev. B 89, 115117 (2014).

[70] A. Rudenko, K. Ladutenko, S. Makarov, and T. E. Itina, Adv. Opt. Mater. 6, 1701153 (2018).

[71] M. A. Green and M. J. Keevers, Prog. Photovoltaics Res. Appl. 3, 189 (1995).

[72] A. Rudenko, J.-P. Colombier, and T. E. Itina, Int. J. Numer Modell. Electron. Networks Devices Fields 31, e2215 (2018).

[73] K. S. Yee, IEEE Trans. Antennas Propag. 14, 302 (1966).

[74] A. Taflove and S. C. Hagness, Computational Electrodynamics: The Finite-Difference Time-Domain Method, 2nd ed. (Artech House, Norwood, MA, 2000).

[75] J. A. Roden and S. D. Gedney, Microwave Opt. Technol. Lett. 27, 334 (2000).

[76] H. M. van Driel, Phys. Rev. B 35, 8166 (1987).

[77] E. J. Yoffa, Phys. Rev. B 21, 2415 (1980).

[78] P. P. Pronko, P. A. VanRompay, C. Horvath, F. Loesel, T. Juhasz, X. Liu, and G. Mourou, Phys. Rev. B 58, 2387 (1998).

[79] J.-L. Déziel, L. J. Dubé, S. H. Messaddeq, Y. Messaddeq, and C. Varin, Phys. Rev. B 97, 205116 (2018).

[80] F. Costache, S. Kouteva-Arguirova, and J. Reif, Appl. Phys. A 79, 1429 (2004).

[81] P. Lalanne, J.-P. Hugonin, H. Liu, and B. Wang, Surf. Sci. Rep. 64, 453 (2009).

[82] A. Y. Nikitin, S. G. Rodrigo, F. García-Vidal, and L. MartínMoreno, New J. Phys. 11, 123020 (2009). 
[83] A. Y. Nikitin, F. J. García-Vidal, and L. Martín-Moreno, Phys. Rev. Lett. 105, 073902 (2010).

[84] A. Abou Saleh, A. Rudenko, L. Douillard, F. Pigeon, F. Garrelie, and J.-P. Colombier, ACS Photonics 6, 2287 (2019).

[85] B. Rethfeld, K. Sokolowski-Tinten, D. von der Linde, and S. I. Anisimov, Phys. Rev. B 65, 092103 (2002).

[86] M. Garcia-Lechuga, D. Puerto, Y. Fuentes-Edfuf, J. Solis, and J. Siegel, ACS Photonics 3, 1961 (2016).

[87] T. Zier, E. S. Zijlstra, and M. E. Garcia, Appl. Phys. A 117, 1 (2014).

[88] A. Rudenko, C. Mauclair, F. Garrelie, R. Stoian, and J.-P. Colombier, Phys. Rev. B 99, 235412 (2019).

[89] G. Tsibidis, E. Stratakis, and K. E. Aifantis, J. Appl. Phys. 111, 053502 (2012).

[90] J. Bonse, S. Baudach, J. Krüger, W. Kautek, and M. Lenzner, Appl. Phys. A 74, 19 (2002).

[91] J. Jia, M. Li, and C. V. Thompson, Appl. Phys. Lett. 84, 3205 (2004).

[92] O. Varlamova, M. Bounhalli, and J. Reif, Appl. Surf. Sci. 278, 62 (2013).
[93] X. Sedao, A. Abou Saleh, A. Rudenko, T. Douillard, C. Esnouf, S. Reynaud, C. Maurice, F. Pigeon, F. Garrelie, and J.-P. Colombier, ACS Photonics 5, 1418 (2018).

[94] P. F. McMillan, Nat. Mater. 1, 19 (2002).

[95] S. Sastry and C. A. Angell, Nat. Mater. 2, 739 (2003).

[96] V. V. Vasisht and S. Sastry, Liq. Polymorphism 152, 463 (2013).

[97] W. D. Luedtke and U. Landman, Phys. Rev. B 40, 1164 (1989). [98] D. Pierce and W. E. Spicer, Phys. Rev. B 5, 3017 (1972).

[99] M. J. Assael, I. J. Armyra, J. Brillo, S. V. Stankus, J. Wu, and W. A. Wakeham, J. Phys. Chem. Ref. Data 41, 033101 (2012).

[100] T. J.-Y. Derrien, J. Krüger, T. E. Itina, S. Höhm, A. Rosenfeld, and J. Bonse, Opt. Express 21, 29643 (2013).

[101] S. Nakamura and T. Hibiya, Int. J. Thermophys. 13, 1061 (1992).

[102] J. Bonse and S. Gräf, Laser Photonics Rev. 14, 2000215 (2020).

[103] Y. Levy, T. J.-Y. Derrien, N. M. Bulgakova, E. L. Gurevich, and T. Mocek, Appl. Surf. Sci. 374, 157 (2016).

[104] I. Prigogine, Science 201, 777 (1978).

[105] R. Lefever, Philos. Trans. R. Soc. A 376, 20170365 (2018). 